\title{
Ba and Eu abundances in M 15 giant stars ${ }^{\star, \star \star}$
}

\author{
C. C. Worley ${ }^{1,2}$, V. Hill ${ }^{1}$, J. Sobeck ${ }^{1,3}$, and E. Carretta ${ }^{4}$ \\ ${ }^{1}$ Laboratoire Lagrange (UMR 7293), Université de Nice Sophia Antipolis, CNRS, Observatoire de la Côte d'Azur, BP 4229, \\ 06304 Nice Cedex 04, France \\ e-mail: ccworley@ast.cam.ac.uk \\ 2 Institute of Astronomy, University of Cambridge, Madingley Rd, Cambridge, CB3 OHA, UK \\ 3 JINA: Joint Institute for Nuclear Astrophysics and the Department of Astronomy and Astrophysics, University of Chicago, \\ 5640 South Ellis Avenue, Chicago, IL 60637, USA \\ ${ }^{4}$ INAF Osservatorio Astronomico di Bologna, via Ranzani 1, 40127 Bologna, Italy
}

Received 13 January 2013 / Accepted 21 February 2013

\begin{abstract}
Aims. We investigate the Ba and Eu abundances for a sample of 63 giant stars in the globular cluster M 15. This is the largest sample of M 15 giant stars for which Ba abundances have been determined and, due to the target selection of the original research programme, the $\mathrm{Ba}$ abundances are complete along the red giant branch.

Methods. Stellar parameters were taken from the previous key study and a microturbulence-surface gravity relation was determined for precise measurement of the Ba line at $6496.898 \AA$, which has a high sensitivity to microturbulence. Element abundances for Ba, $\mathrm{La}, \mathrm{Eu}, \mathrm{Ca}, \mathrm{Ni}$ and $\mathrm{Fe}$ were calculated using spectrum synthesis and equivalent widths techniques.

Results. A bimodal distribution in $\mathrm{Ba}, \mathrm{Eu}$ and $\mathrm{La}$ abundances was found within the sample. The low $\mathrm{Ba}, \mathrm{Eu}, \mathrm{La}$ mode had mean abundances of $\langle[\mathrm{Ba} / \mathrm{H}]\rangle=-2.41 \pm 0.16,\langle[\mathrm{Eu} / \mathrm{H}]\rangle=-1.80 \pm 0.08$ and $\langle[\mathrm{La} / \mathrm{H}]\rangle=-2.19 \pm 0.13$ while the high Ba, Eu, La mode had mean abundances of $\langle[\mathrm{Ba} / \mathrm{H}]\rangle=-2.00 \pm 0.16,\langle[\mathrm{Eu} / \mathrm{H}]\rangle=-1.65 \pm 0.13$ and $\langle[\mathrm{La} / \mathrm{H}]\rangle=-1.95 \pm 0.11$.

Conclusions. Both modes are indicative of a pollution scenario dominated by the $r$-process, hence contributions from explosive nucleosynthesis of massive stars. There may be evidence of further enhancement by another heavy element process and of potential anticorrelations in $\mathrm{Na}-\mathrm{O}$ for both modes indicating a complex formation and evolution history for $\mathrm{M} 15$.
\end{abstract}

Key words. stars: abundances - galaxies: groups: individual: M 15

\section{Introduction}

The globular clusters of the Milky Way provide a wealth of information regarding the evolution of stars and the galaxy. Recent advances in technology and analysis techniques have revealed that many globular clusters show evidence of multiple stellar populations through photometry and chemical abundance distributions. A recent review of these discoveries is given in Gratton et al. (2012). Disentangling the different stellar populations by their chemical signatures allows astronomers to identify the pollution and evolution mechanisms at work in these complex stellar systems.

The origins of many of the light $(Z \leq 30)$ and heavy $(Z>30)$ elements can be attributed to key stages in stellar evolution, whereby each stage produces unique chemical signatures, depending on the mass and metallicity of the progenitor star. The contributions from the different stages of stellar evolution are determined by identifying the quantity and distribution of these elements in the observed stellar population and considering them in combination with the knowledge of the sites of nucleosynthesis in which the elements are produced.

* Based on observations collected at the European Organisation for Astronomical Research in the Southern Hemisphere, Chile. Proposal ID: 080.B-0784(A).

$\star \star$ Tables 1,3 , and 11 are available in electronic from at http://www. aanda.org
The metal-poor globular cluster M 15, with $[\mathrm{Fe} / \mathrm{H}]=$ $-2.31 \pm 0.06$ dex (Carretta et al. 2009b), has proven to be an interesting case for study due in particular to star-to-star variations in $[\mathrm{Eu} / \mathrm{H}]$ and $[\mathrm{Ba} / \mathrm{H}]$ that have been found. Eu is predominantly an $r$-process element, formed due to the rapid capture of neutrons by a seed nuclei $(\mathrm{Fe})$ in a high density neutron environment. The key stellar evolution nucleosynthesis site for the $r$-process is explosive nucleosynthesis in massive stars, such as in Type II supernovae (Sneden et al. 2008), which is also the key production site for the $\alpha$ elements $(\mathrm{O}, \mathrm{Mg}, \mathrm{Si}, \mathrm{Ca}, \mathrm{Ti} \ldots)$ (Edvardsson et al. 1993). Hence comparing Eu and $\alpha$ elements may be used to probe the mass-range of the supernovae progenitors. $\mathrm{Ba}$ is a neutron capture element that can be produced by the $r$-process, but is predominantly produced in the solar system material by the $s$-process, which is the slow capture of neutrons by a seed nuclei. The typical nucleosynthesis site for the $s$-process is within thermally pulsing asymptotic giant branch (AGB) stars (Busso et al. 2001) and it is possibly also significant in massive fast-rotating stars (Iliadis 2007). Hence the comparison of $s$ - and $r$-process element abundances also provides a means by which to discriminate between the contributions from these very different nucleosynthesis sites.

The study of Sneden et al. (1997, hereafter S97) investigated the abundances of both light $(\mathrm{O}, \mathrm{Na}, \mathrm{Mg}, \mathrm{Al}, \ldots)$ and heavy $(\mathrm{Ba}$, $\mathrm{Eu})$ elements in a sample of 18 bright giants in M 15. Anticorrelations typical to globular clusters were observed between $\mathrm{Na}$ and $\mathrm{O}$, and between $\mathrm{Al}$ and $\mathrm{Mg}$. Fe, $\mathrm{Ca}$ and $\mathrm{Si}$ were found to 
have uniform abundances for the sample. Eu and Ba were found to be strongly correlated with a possible bimodal distribution of both abundances, and to each have a large spread in values on the order of 0.6 dex. But no obvious correlation was found between these heavy elements and the light elements. Hence it was concluded that $\mathrm{Ba}$ and $\mathrm{Eu}$ had a primordial origin due to Type II supernova of a stellar mass high enough to produce $r$-process elements but not sufficiently high to produce $\alpha$ elements, hence the lack of any correlation between those two types of elements.

This work was then followed by two further studies, Sneden et al. (2000a, hereafter S00a) and Sneden et al. (2000b, hereafter S00b). In the former study three of the stars at the tip of the giant branch analysed in Sneden et al. (1997) were re-observed to investigate further the neutron-capture abundances in these stars. The stars were chosen as having similar stellar parameters but very different Eu and Ba abundances as per Sneden et al. (1997). These differences were confirmed and the heavy element signatures that were derived closely followed that produced primarily by the $r$-process.

In the latter study a further 31 giant stars in M 15 were analysed to investigate the proton-capture $(\mathrm{Na}), \alpha$-capture $(\mathrm{Ca}, \mathrm{Si}$, $\mathrm{Ti})$ and neutron-capture (Ba) element abundances. It was found that there was a large range in $\mathrm{Na}$ abundances, which was attributed to proton-capture on Ne occurring internally to the observed stars, as well as a large range in Ba comparable to that found in Sneden et al. (1997) confirming that result and the bimodal distribution of $\mathrm{Ba}$. Eu abundances could not be measured for this sample.

Otsuki et al. (2006, hereafter O06) investigated the heavy element abundances of seven giant stars in M 15 that had also been analysed in S97. They found $\mathrm{Fe}$ and Eu abundances in agreement with S97 and extended the analysis to Zr, Y and La. They found that La correlated with Eu, as expected, but observed an anti-correlation of $\mathrm{Zr}$ and $\mathrm{Y}$ with Eu. From this they concluded that while the $\mathrm{La}$ (and $\mathrm{Ba}$ ) correlation with Eu implies a primary contribution of neutron-capture elements by an $r$-process only mechanism (e.g. explosive nucleosynthesis within massive stars) this cannot also explain the anticorrelation of $\mathrm{Zr}$ and $\mathrm{Y}$ with $\mathrm{Eu}$. They argue that some other process enriched the interstellar medium in M 15 in light neutron-capture elements before the enrichment of the heavy neutron-capture elements by supernova.

Preston et al. (2006, hereafter P06) made an investigation of six red horizontal branch (RHB) stars in M 15. Both light and heavy element abundances were determined, for the M 15 stars similar results regarding the evidence of star-to-star scatter in the heavy element abundances were found. Also noted was that the RHB stars of M 15 were found to be $\sim 0.2$ dex more metal-poor than red giant branch (RGB) stars in the same cluster.

Most recently, a detailed investigation into neutron-capture element abundances in giant stars in M 15 was carried out by Sobeck et al. (2011, hereafter Sk11). The three RGB stars of S00a and the six RHB stars of P06 were re-analysed in a consistent manner in order to investigate the nature of the heavy element abundances in M 15 giant stars, and to investigate the metallicity offset between the RHB and RGB as observed in P06. The abundances for 40 species of both light and heavy elements were derived for these nine stars. As had been observed previously the abundances of proton-capture elements showed no correlation with the abundances of the neutron capture elements. In particular a metallicity offset between the RHB and RGB was found, and the large spread in $\mathrm{Ba}$ and $\mathrm{Eu}$ was confirmed. However the bimodal distribution of $\mathrm{Ba}$ and $\mathrm{Eu}$ that was proposed in Sneden et al. (1997) was not observed. The analysis concluded that the heavy neutron-capture element abundances agreed well with a scaled-solar $r$-process distribution, but the light neutron-capture element abundances did not agree with either a scaled-solar $s$ - or $r$-process distribution. This was therefore evidence of some other nucleosynthetic process, under the umbrella of the Lighter Element Primary Process (LEPP), at work.

The largest sample of M 15 stars analysed spectroscopically to-date was carried out by Carretta et al. (2009b, hereafter C09) as part of an analysis of light element abundances for stellar samples within 15 galactic globular clusters observed using FLAMES/GIRAFFE. Fe, $\mathrm{Na}$ and $\mathrm{O}$ abundances were investigated for 84 stars in M 15. In total the research programme obtained homogeneously determined abundances for $\mathrm{Na}$ and $\mathrm{O}$ for 1235 stars in 19 globular clusters. An anti-correlation between $\mathrm{Na}$ and $\mathrm{O}$ was observed for all the clusters, including $\mathrm{M} 15$. This provided evidence of a pristine first generation of stars $(\sim 30 \%$ of the observed cluster stars) and a second generation that had formed from material enriched in H-burning products by nowextinct massive stars of the first stellar generation. Carretta et al. (2009a) followed up this study with high resolution observations of 200 red giants across $17 \mathrm{GCs}$, for which 13 stars were observed in M 15 from the C09 sample.

Of particular relevance is the study undertaken in D'Orazi et al. (2010, hereafter DO10) which investigated Ba abundances in GC stars directly from the observations of C09. This study measured $\mathrm{Ba}$ for 1200 stars across the $15 \mathrm{GCs}$, including measurements for 57 stars in the M 15 sample. M 15 was the only sample for which they found a large variation in the Ba abundances between the stars confirming the large variation found in S97 and S00b.

The study carried out here seeks to extend the sample of giant stars in M 15 for which heavy neutron-capture element abundances have been determined and consider their distribution in M 15 in light of the previous studies.

Section 2 describes the observations and presents the stellar parameters for the dataset under analysis. Section 3 describes the determination of the stellar parameters as per Carretta et al. (2009b) and the treatment of the microtubulence, $\xi$, in this study. Section 4 describes the determination of the chemical abundances for this sample. Section 5 present the results of the chemical abundance analysis. Section 6 discusses possible scenarios based on the results. Section 7 concludes the paper.

\section{Observations}

Observations of 63 RGB stars in M 15 were taken using the HR14A setup of GIRAFFE on the VLT in November 2009. The spectra were wavelength calibrated as from the ESO pipeline and sky-subtraction was performed using IRAF. Normalisation and radial velocity determination were performed using tools developed for the AMBRE Project (Worley et al. 2012). The signalto-noise $(\mathrm{S} / \mathrm{N})$ ranged from 50 to 170 across the sample.

These targets were taken from $\mathrm{C} 09$ in which the stellar parameters of effective temperature $\left(T_{\text {eff }}\right)$, surface gravity $(\log g)$ and metallicity $([\mathrm{Fe} / \mathrm{H}])$ as well as individual chemical abundances for $\mathrm{Na}$ and $\mathrm{O}$ were obtained for a total sample of 84 stars in M 15. Table 1 lists the star ID and stellar parameters of the 63 stars from C09 that were analysed here as taken from Table 3 in $\mathrm{C} 09$.

The DO10 sample was also a subsample of C09. Of the 57 DO10 stars, 38 were also observed for this study. D010 could not analyse the complete $\mathrm{C} 09$ sample for $\mathrm{Ba}$ abundances as not all the stars were observed using the HR13 grating, in which the Ba spectral feature at $6141.73 \AA$ is present. 

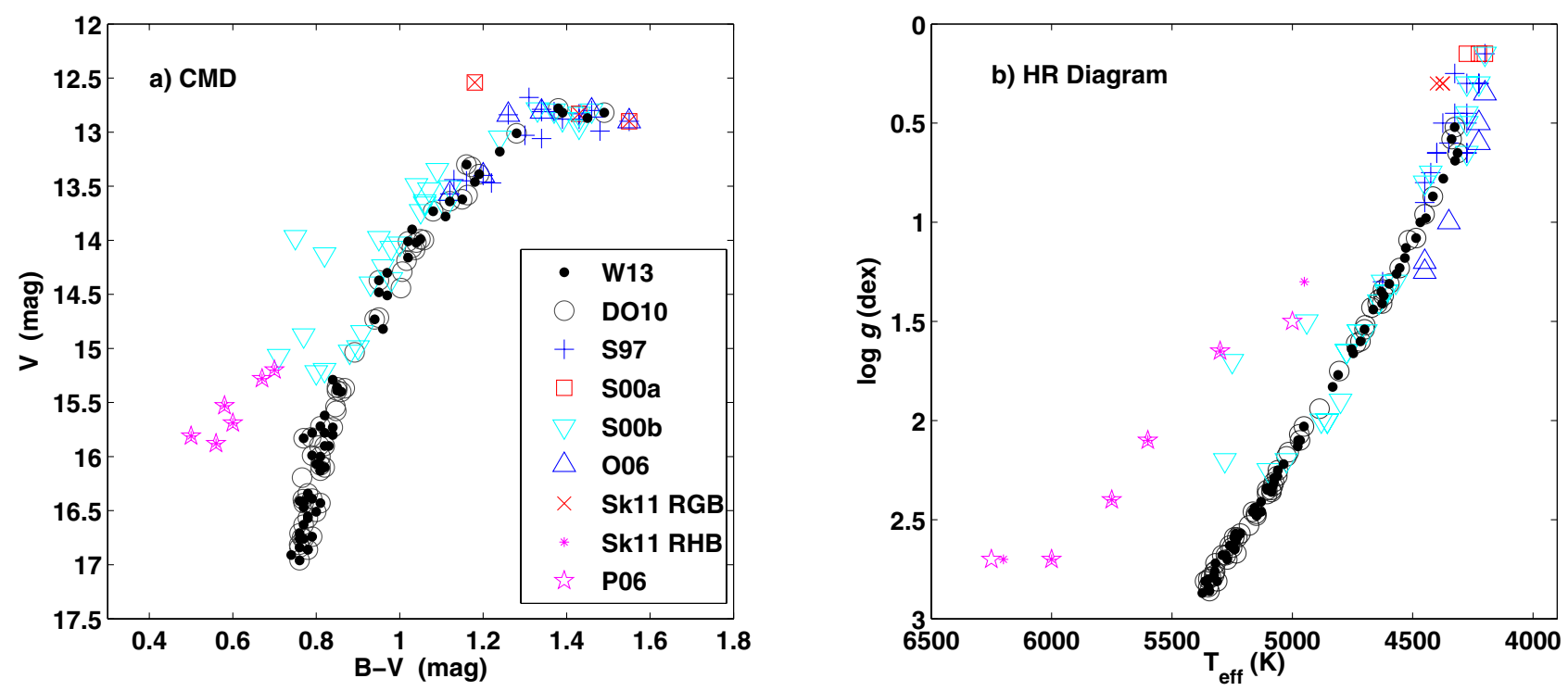

Fig. 1. CMD and HR diagram of the key samples of giant stars in M 15 as per the legend. The colours and parameters for W13 and DO10 both come from $\mathrm{C} 09$.

The colour-magnitude diagram (CMD) and HertsprungRussell (HR) diagram of the 63 stars from C09 that have been observed and analysed here are shown in Fig. 1. The locations of stars from previous key studies of M 15 are also shown for comparison including the DO10 subsample of C09.

The wavelength range of the HR14 grating covers from $\sim 6308 \AA$ to $\sim 6700 \AA$, which includes the key neutroncapture features of Ba II at $6496.898 \AA$, La II at $6390.4770 \AA$, and Eu II at $6437.640 \AA$ and $6645.127 \AA$. At this resolution of $R \sim 14500$ these three features are observable although the Eu II features are weak. The astrophysical quantities associated with these lines, as well as those for the other lines measured in this study, are listed in Table 2. Table 3 lists the hyperfine structure decomposition for the Ba II, La II and Eu II spectral lines as well as the references from which the atomic information was obtained upon which the calculations were based.

\section{Stellar parameters}

The stellar parameters for each of the stars observed here had been determined in C09. The $T_{\text {eff }}$ was determined in a two step process whereby a relation between $T_{\text {eff }}$ and the $V$ (or $K$ ) magnitude was developed for the cluster using $V-K$ photometry and the Alonso et al. (1999) calibration. This relation was then used to calculate the $T_{\text {eff }}$ for each star in the sample. The $\log g$ was determined using $T_{\text {eff }}$, the apparent magnitude, the distance modulus and bolometric corrections from Alonso et al. (1999). The $\xi$ was determined by eliminating any trend of the abundance of iron with expected line strength based on the equivalent width measurement of Fe I lines. See C09 and references therein for more detailed descriptions of the methods employed.

However, as can be noted from Table 1, the resulting microturbulence velocities for the C09 M 15 sample range from 0.03 to $2.7 \mathrm{~km} \mathrm{~s}^{-1}$ which is a very wide and rather implausible range for this sample of giant stars. This wide range most probably results from too few and too weak Fe lines being available in the spectral range used in the C09 study for this very metalpoor cluster. These $\xi$ values anyway have almost no impact on the resulting abundances of $\mathrm{Fe}, \mathrm{O}$ and $\mathrm{Na}$ investigated in $\mathrm{C} 09$, since the lines analysed were on the linear part of the curve of
Table 2. List of $\mathrm{Fe}, \mathrm{Ca}, \mathrm{Ni}, \mathrm{Ba}, \mathrm{Eu}$, and $\mathrm{La}$ lines used in this analysis.

\begin{tabular}{|c|c|c|c|c|c|c|}
\hline Wavelength $(\AA)$ & Species & $\chi(\mathrm{eV})$ & $\log g f$ & Reference & Method & $\mathrm{Sol}^{*}$ \\
\hline 6439.0700 & 20.0 & 2.526 & 0.470 & NIST & SS & 6.41 \\
\hline 6471.6600 & 20.0 & 2.526 & -0.590 & NIST & SS & " \\
\hline 6318.0171 & 26.0 & 2.453 & -1.804 & NIST & EW & 7.54 \\
\hline 6322.6851 & 26.0 & 2.588 & -2.426 & NIST & EW & " \\
\hline 6335.3304 & 26.0 & 2.198 & -2.177 & NIST & EW & " \\
\hline 6336.8239 & 26.0 & 3.686 & -0.856 & NIST & EW & " \\
\hline 6344.1487 & 26.0 & 2.433 & -2.923 & NIST & EW & " \\
\hline 6355.0286 & 26.0 & 2.845 & -2.291 & NIST & EW & " \\
\hline 6393.6009 & 26.0 & 2.433 & -1.576 & NIST & EW & " \\
\hline 6408.0179 & 26.0 & 3.686 & -1.018 & NIST & EW & " \\
\hline 6411.6489 & 26.0 & 3.654 & -0.718 & NIST & EW & \\
\hline 6421.3504 & 26.0 & 2.279 & -2.027 & NIST & EW & " \\
\hline 6430.8460 & 26.0 & 2.176 & -2.006 & NIST & EW & " \\
\hline 6475.6240 & 26.0 & 2.559 & -2.942 & NIST & EW & " \\
\hline 6481.8699 & 26.0 & 2.279 & -2.984 & NIST & EW & $"$ \\
\hline 6518.3667 & 26.0 & 2.832 & -2.298 & NIST & EW & " \\
\hline 6575.0154 & 26.0 & 2.588 & -2.710 & NIST & EW & " \\
\hline 6592.9134 & 26.0 & 2.728 & -1.473 & NIST & EW & " \\
\hline 6593.8701 & 26.0 & 2.433 & -2.422 & NIST & EW & " \\
\hline 6432.6800 & 26.1 & 2.891 & -3.500 & NIST & EW & " \\
\hline 6456.3760 & 26.1 & 3.903 & -2.190 & NIST & EW & $"$ \\
\hline 6516.0530 & 26.1 & 2.891 & -3.372 & NIST & EW & "' \\
\hline 6643.6300 & 28.0 & 1.675 & -2.300 & NIST & SS & 6.29 \\
\hline 6496.8980 & Ba II & 0.604 & -0.407 & $\mathrm{D} 12^{a}$ & SS & 2.25 \\
\hline 6390.4770 & La II & 0.321 & -1.410 & $\mathrm{~L}^{01 \mathrm{a}^{a}}$ & SS & 1.25 \\
\hline 6437.6400 & $\mathrm{Eu} \mathrm{II}$ & 1.320 & -0.320 & $\mathrm{~L}^{0} 01 b^{b}$ & SS & 0.60 \\
\hline 6645.1270 & Eu II & 1.380 & 0.120 & $\mathrm{~L}^{0} 1 \mathrm{~b}^{c}$ & SS & \\
\hline
\end{tabular}

Notes. Includes the associated excitation potential $(\chi), \log g f$, the method of analysis used: spectrum synthesis (SS) or equivalent width curve of growth (EW), and reference solar abundances.

${ }^{(*)}$ Solar reference abundances taken from Lodders (2003). ${ }^{(a)}$ Davidson et al. (1992); ${ }^{(b)}$ Lawler et al. (2001a); ${ }^{(c)}$ Lawler et al. (2001b).

growth. On the other hand, the $\mathrm{Ba}$ line under analysis here is strong enough for the microturbulence to play a role in desaturating the lines.

The sensitivity of the $6496.898 \AA$ Ba line to $\xi$ is clearly shown in the error analysis in Table 5 . In a preliminary analysis 
Table 4. Stellar parameters from C09 for 13 stars for which the equivalent widths of Fe I and Fe II lines were measured and/or were analysed in previous studies.

\begin{tabular}{|c|c|c|c|c|c|c|c|c|c|c|c|c|c|}
\hline Star ID & Other ID & $T_{\text {eff }}$ & $\log g$ & {$[\mathrm{~A} / \mathrm{H}]$} & $\xi$ & $\xi_{\mathrm{W} 13}$ & {$[\mathrm{Fe} \mathrm{I} / \mathrm{H}]$} & $\sigma$ & $N^{\#}$ & {$[\mathrm{Fe} \mathrm{II} / \mathrm{H}]$} & $\sigma$ & $N$ & Other studies \\
\hline 40825 & K386 & 4313 & 0.65 & -2.33 & 2.25 & 2.25 & -2.40 & 0.10 & $35(22)$ & -2.37 & - & 2 & S97, S00b, Ot06 \\
\hline 4099 & K341 & 4324 & 0.69 & -2.32 & 2.03 & 2.30 & -2.40 & 0.11 & 17 & -2.34 & - & 2 & S97, S00a, S00b, Sk11 \\
\hline 43788 & K825 & 4325 & 0.52 & -2.36 & 2.40 & 2.25 & -2.32 & 0.12 & 17 & -2.37 & - & 2 & S97, S00b \\
\hline 31914 & K757 & 4338 & 0.58 & -2.20 & 2.90 & 2.10 & -2.27 & 0.11 & 17 & -2.33 & - & 2 & S97, S00b \\
\hline 41287 & K702 & 4373 & 0.78 & -2.36 & 1.10 & 2.10 & -2.41 & 0.13 & 17 & -2.37 & - & 2 & S97 \\
\hline 34519 & & 4416 & 0.87 & -2.38 & 1.62 & 2.00 & -2.44 & 0.10 & $23(9)$ & -2.32 & - & 2 & \\
\hline 34995 & & 4468 & 1.00 & -2.34 & 0.79 & 2.10 & -2.41 & 0.13 & 15 & -2.40 & - & 2 & \\
\hline 3137 & K387 & 4486 & 1.08 & -2.35 & 2.27 & 2.00 & -2.38 & 0.12 & 16 & -2.32 & - & 2 & S97 \\
\hline 26751 & K709 & 4533 & 1.18 & -2.44 & 1.40 & 2.02 & & & & & & & S00b \\
\hline 38678 & K288 & 4554 & 1.23 & -2.35 & 1.28 & 2.00 & -2.47 & 0.11 & $20(9)$ & -2.38 & - & 2 & SOOb \\
\hline 2792 & $\mathrm{~K} 255$ & 4567 & 1.26 & -2.32 & 1.50 & 2.20 & -2.47 & 0.13 & 11 & -2.37 & 0.14 & 3 & SO0b \\
\hline 34961 & & 4627 & 1.41 & -2.32 & 1.85 & 2.00 & -2.46 & 0.13 & $22(14)$ & -2.46 & 0.09 & 3 & \\
\hline 38329 & & 4664 & 1.44 & -2.37 & 0.86 & 1.80 & -2.41 & 0.12 & 11 & -2.28 & - & 2 & \\
\hline
\end{tabular}

Notes. The mean and standard deviation and the number of lines measured for Fe I and Fe II are listed. The standard deviation is given only for measurements of $N>2{ }^{(\#)}$ The numbers in brackets are the number of equivalent widths obtained from C09.
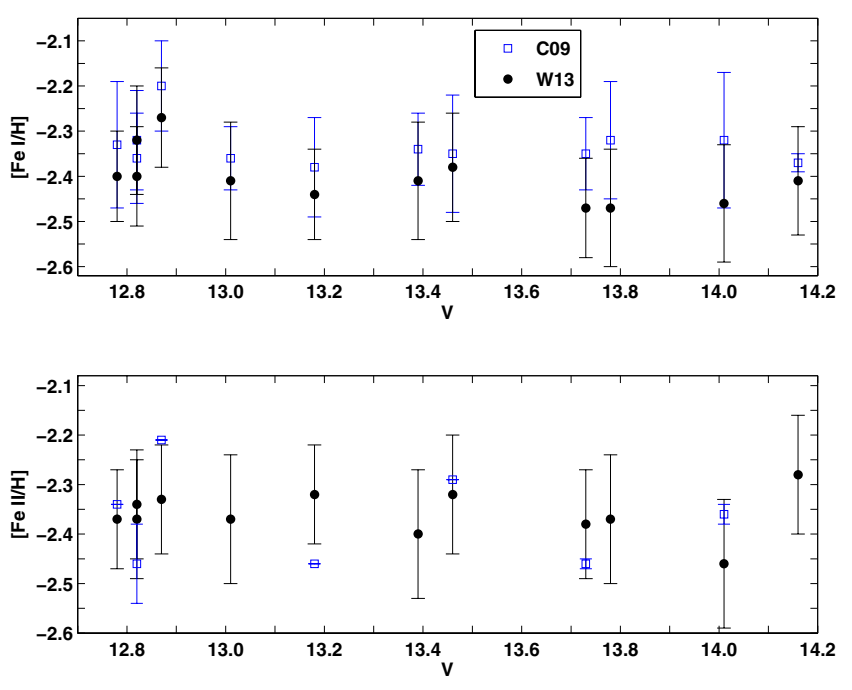

Fig. 2. Comparison of $[\mathrm{Fe} \mathrm{I} / \mathrm{H}]$ and $[\mathrm{Fe} \mathrm{II} / \mathrm{H}]$ values from $\mathrm{C} 09$ and the values determined here by equivalent width measurement. The C09 $[\mathrm{Fe} \mathrm{I} / \mathrm{H}]$ are those used as input for the stellar atmosphere models.

using the $\xi$ values determined in $\mathrm{C} 09$, a clear trend of Ba abundance with $\xi$ was found. This is a typical problem when dealing with the measurement of strong lines such as those of $\mathrm{Ba}$ in giants. In SOOb a constant $\xi$ of $2.0 \mathrm{~km} \mathrm{~s}^{-1}$ was assumed while Sk11 treated $\xi$ so as to eliminate any trend for the strong lines.

For this sample we therefore re-determined $\xi$ using the available Fe I lines as listed in Table 2. Hence the equivalent widths for Fe I and Fe II lines were measured for those stars in which a sufficient number of unblended lines could be identified. For the majority of stars the combination of low $\mathrm{S} / \mathrm{N}$ and high temperatures meant a insufficient sample of Fe lines could be identified.

Table 4 lists the C09 parameters for 13 stars of which 12 had a sufficient sample of Fe lines that could be used to determine the microturbulence. The microturbulence determined in this analysis $\left(\xi_{\mathrm{W} 13}\right)$ with the mean $[\mathrm{Fe} \mathrm{I} / \mathrm{H}]$, mean $[\mathrm{Fe} \mathrm{II} / \mathrm{H}]$, associated standard deviations and number of lines measured are also listed. For four of the spectra the equivalent widths of Fe I lines measured in C09 for the corresponding stars were also used and the number of lines for each are also indicated. Hence for each of these 12 stars the equivalent widths of all possible Fe I and Fe II lines were measured and abundances determined using the curve of growth analysis routine, abfind, in the one-dimensional, local thermodynamic equilibrium (LTE) radiative transfer code, MOOG (Sneden 1973). Stellar models for each star were generated at the C09 stellar parameters using the Kurucz 1995 stellar model grid and the ATLAS9 ${ }^{1}$ model atmosphere calculation programme.

Eight of this sample of stars had also been analysed in previous studies, as well a ninth star for which Fe I lines could not be measured here. The papers associated with each star are also listed in Table 4.

Figure 2 compares the mean $[\mathrm{Fe} \mathrm{I/H}]$ and mean $[\mathrm{Fe} \mathrm{II/H]} \mathrm{val-}$ ues measured here with those measured in $\mathrm{C} 09$ for the 12 stars, although in C09 Fe II lines could only be measured for 6 of the current sample. The errorbars on the W13 measurements of Fe II lines come from the uncertainty on the measurement of the Fe I lines in the corresponding star, as the dispersion around the Fe I lines is a fair representation of the observational scatter for the measurement of weak lines in these spectra. Thus Fig. 2 shows that there is agreement within $1 \sigma$ between $\mathrm{C} 09$ and $\mathrm{W} 13$ for $[\mathrm{Fe} \mathrm{I} / \mathrm{H}]$ and $[\mathrm{Fe} \mathrm{II} / \mathrm{H}]$.

Based on the $\xi_{\mathrm{W} 13}$ derived for these 12 stars a $\xi$ - $\log g$ relation was determined as the linear best fit to the 12 data points. This relation was then used to provide a $\xi_{\mathrm{W} 13}$ value for the remaining stars in the sample calculated from their $\log g$ value. Figure 3 shows the $\xi$ and $\log g$ values as determined in C09 and in this study (W13) with the $\xi$-log $g$ relation and uncertainty limits determined in Kirby et al. (2009, hereafter K09). The linear best fit to the $\xi_{\mathrm{W} 13}$ values is also shown, extended across the range of $\log g$ values for this sample. The equations for the K09 relation and relation derived here are as follows:

$\xi_{\mathrm{K} 09}=(2.13 \pm 0.05)-(0.23 \pm 0.03) \log g$,

$\xi_{\mathrm{W} 13}=2.386-0.3067 \log g$.

This sample is tailored specifically to these mono-metallicity stars while the K09 relation was derived from a sample of stars covering a range of metallicities. However the relations are in reasonably good agreement and in the expected range for giant stars.

1 http://kurucz.harvard.edu/programs.html 


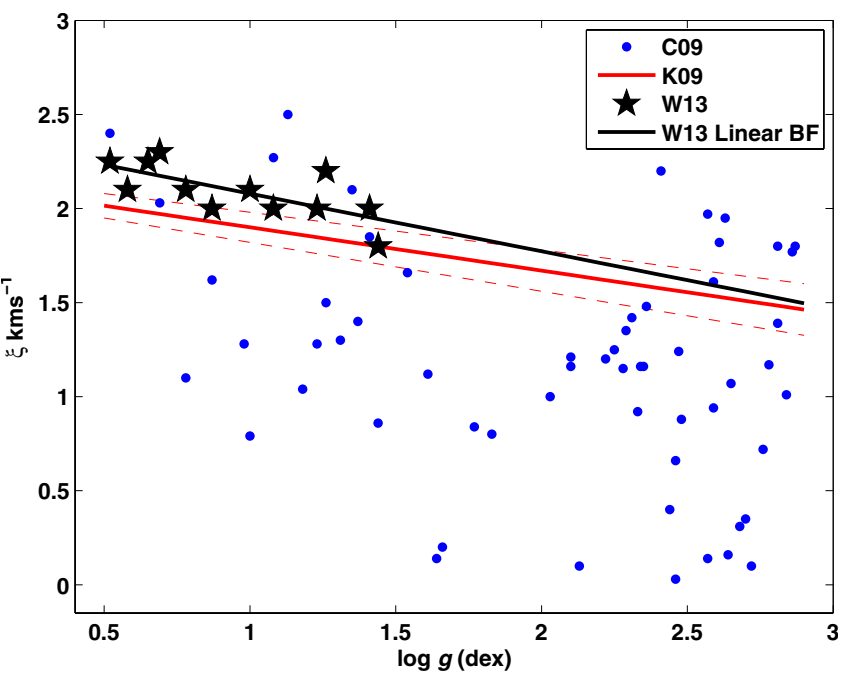

Fig. 3. Comparison of $\mathrm{C} 09 \xi$ and $\log g$ values with the $\xi-\log g$ relation from $\mathrm{K} 09$, the $\xi$ values determined here for the sample of 12 stars and the resulting $\xi$ - $\log g$ relation that was derived.

\section{Determination of stellar abundances}

As mentioned above the iron abundances based on $\mathrm{Fe} I$ and Fe II lines were determined using equivalent widths and curve-of-growth analysis in MOOG. For the determination of the abundances for $\mathrm{Ca}, \mathrm{Ni}, \mathrm{Ba}, \mathrm{La}$ and $\mathrm{Eu}$ the techniques of spectrum synthesis were employed, again using MOOG and the Kurucz 1995 stellar models interpolated to the C09 parameters but now with the $\xi_{\mathrm{W} 13}$ values. The most recent laboratory $\log g f$ s were obtained, as listed in Table 2 and the hyperfine structure for the $\mathrm{Ba}$ II, La II and Eu II were taken into account as per the decomposition in Table 3 based on the most recent atomic information (Rutten 1978; Davidson et al. 1992; Lawler et al. 2001a,b).

\subsection{Comparison stars}

As shown in Table 4 there are nine stars in this sample that have been analysed in previous studies. Figure 4 shows the range in values obtained for these stars for their stellar parameters and individual element abundances.

The stellar parameters of $T_{\text {eff }}$ are mostly within $50 \mathrm{~K}$ in terms of agreement between the studies (Fig. 4a). However for four of the stars the spread in $\log g$ is on the order of 0.5 dex (Fig. 4b) and $\xi$ is particularly spread out $\left(\sim 0.3 \mathrm{~km} \mathrm{~s}^{-1}\right)$ between the studies for the high luminosity, low $\log g$ stars (Fig. 4c), which is reflective of the difficulty of deriving parameters for these types of stars. These differences reflect the degree of variation in stellar parameters that can be derived for the same star using different observations, measurements and analysis techniques. For example the three hottest stars have parameters from C09 and S00b only. For S00b, if the stars were not from S97, then the parameters were inferred from photometry- $T_{\text {eff }}, \log g$ relations. Hence the $\mathrm{C} 09$ parameters and S00b parameters are in good agreement for $T_{\text {eff }}$ and $\log g$, compared to the next two coolest stars which have C09 and S97 parameters. S97 derived parameters spectroscopically and while the $T_{\text {eff }}$ agree reasonably well with C09 the $\log g$ do not. However the good agreement in $[\mathrm{Fe} / \mathrm{H}]$ between the studies is seen in Fig. 4c.

Figure $4 \mathrm{~d}$ to $\mathrm{h}$ compare the abundances for $\mathrm{Ca}, \mathrm{Ni}, \mathrm{Ba}$ and $\mathrm{Eu}$ derived here with the abundances from the other studies for the comparison stars. There is reasonable agreement between the studies. $\mathrm{Ca}$ in particular shows good agreement between the
Table 5. Variations in measured abundances with changes in stellar atmospheric parameters for the sample of Fe I and Fe II lines, by equivalent width measurement, and the other key lines used in this study.

\begin{tabular}{lccc}
\hline \hline & $\Delta T_{\text {eff }}$ & $\Delta \log g$ & $\Delta \xi$ \\
& $+50 \mathrm{~K}$ & $+0.5 \mathrm{dex}$ & $-0.25 \mathrm{~km} \mathrm{~s}^{-1}$ \\
\hline$\Delta[\mathrm{Fe} \mathrm{I} / \mathrm{H}]$ & 0.10 & -0.06 & 0.06 \\
$\Delta[\mathrm{Fe} \mathrm{II} / \mathrm{H}]$ & -0.02 & 0.18 & 0.01 \\
$\Delta[\mathrm{Ca} \mathrm{I} / \mathrm{Fe}](6439.07)$ & 0.10 & -0.08 & 0.14 \\
$\Delta[\mathrm{Ca} \mathrm{I} / \mathrm{Fe}](6471.66)$ & 0.06 & -0.06 & 0.02 \\
$\Delta[\mathrm{Ni} \mathrm{I} / \mathrm{Fe}]$ & 0.10 & -0.03 & 0.07 \\
$\Delta[\mathrm{Ba} \mathrm{II} / \mathrm{Fe}]$ & 0.05 & 0.17 & 0.23 \\
$\Delta[\mathrm{Eu} \mathrm{II} / \mathrm{Fe}](6437.64)$ & 0.00 & 0.16 & 0.08 \\
$\Delta[\mathrm{Eu} \mathrm{II} / \mathrm{Fe}](6645.13)$ & 0.00 & 0.17 & 0.00 \\
$\Delta[\mathrm{La} \mathrm{II} / \mathrm{Fe}]$ & 0.03 & 0.18 & 0.01 \\
\hline
\end{tabular}

studies at a reasonably constant absolute abundance. Ni is constant in abundance for this study but the three comparisons to two other studies show some variation. The range in abundance values between the stars for $\mathrm{Ba}$ and $\mathrm{Eu}$ is obvious already for this small sample of stars. For the chemical abundances the disagreement between studies is greatest for the high luminosity, reflecting the uncertainties in stellar parameters and chemical abundance determination for stars of low $\log g$. There may also be uncertainties in the model atmospheres (e.g. sphericity effects) and in non-local thermodynamic equilibrium effects associated with cool giants of low gravity.

Table 5 gives the expected variations in the derived abundances for a $\Delta T_{\text {eff }}=50 \mathrm{~K}, \Delta \log g=+0.5 \mathrm{dex}$ and $\Delta \xi=$ $-0.25 \mathrm{~km} \mathrm{~s}^{-1}$ for the light and heavy element lines being analysed in this study. The neutral species show greater sensitivity to changes in $T_{\text {eff }}$, while the ionised species show greater sensitivity to changes in $\log g$ as expected. The Ca I line at $6471.66 \AA$ and the Ba II line both show a sensitivity to changes in $\xi$, in particular the $\mathrm{Ba}$ II line which varies by 0.23 dex, as is expected for strong lines.

The possible effects of departures from LTE on the Ba spectral feature at $6496 \AA$ have been previously examined by Short \& Hauschildt (2006) and Andrievsky et al. (2009). Short \& Hauschildt (2006) analysed the non-LTE sensitivity of the Ba II 6496 transition for a variety of different stellar atmospheric model and line transfer combinations in a typical halo giant (with $T_{\text {eff }}=4800 \mathrm{~K}$ and $\log g=1.5$ ). By and large, they found a metallicity dependence of the non-LTE effect with line strength (explicitly, a non-LTE weakening of transitions for the most metal-deficient models and conversely, a non-LTE strengthening of lines for the least metal-poor models). Yet, in particular for the 6496 transition, when the data for full non-LTE model/transfer are compared to those for LTE model/transfer, the magnitude of the change was relatively small on the resultant $\mathrm{Ba}$ abundance (with $\mid \log W_{\lambda}$ (NLTEFull) $-\log W_{\lambda}$ (LTE) $\mid \leq 0.03$ ).

On the other hand, Andrievsky et al. (2009) employed a single LTE atmospheric model with non-LTE radiative transfer to study the non-LTE effect on Ba II lines as a function of both metallicity and temperature in extremely metal-poor giants. Their results mostly confirm those of Short \& Hauschildt (2006) (a correlation between metallicity and non-LTE departure was established with the largest effect seen for warm, metalpoor stars). Specifically for the Ba II $6496 \AA$ feature, they found a maximum negative correction factor (NLTE-LTE) of $\approx-0.1$ for a giant with $[\mathrm{Fe} / \mathrm{H}]=-2.0$ and $T_{\text {eff }}=4700 \mathrm{~K}$ and a maximum positive (NLTE-LTE) correction of $\approx-0.13$ for a giant with $[\mathrm{Fe} / \mathrm{H}]=-2.5$ and $T_{\text {eff }}=5500 \mathrm{~K}$. Andrievsky et al. (2009) 

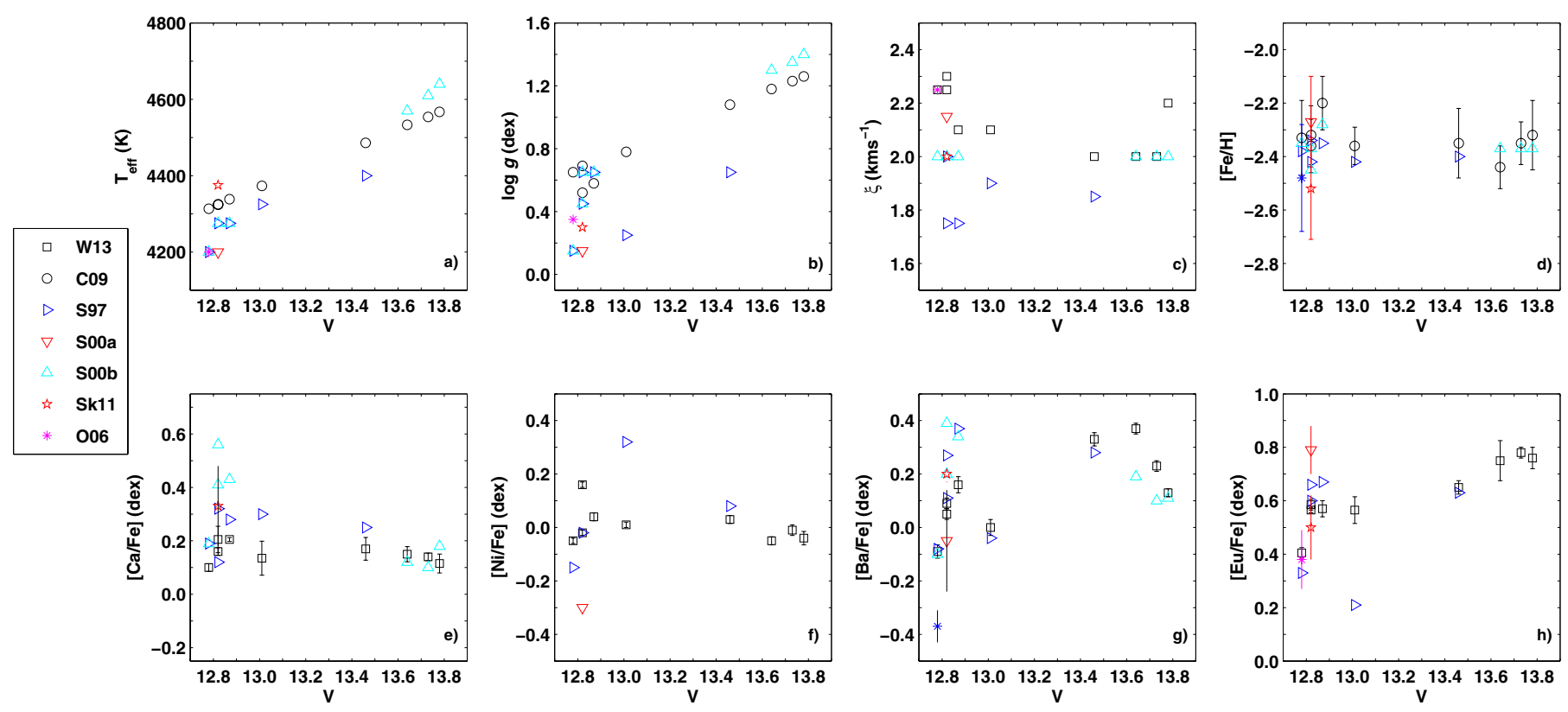

Fig. 4. Stellar parameters and chemical abundances against $V$ magnitude for the 9 comparison stars in the M 15 sample. The values from C09, W13, S97, S00a, S00b, O06 and Sk11 are shown: a) $T_{\text {eff }}$ b) $\log g$; c) $\xi$; d) $[\mathrm{Fe} / \mathrm{H}]$; e) $\left.\left.[\mathrm{Ca} / \mathrm{H}] ; \mathbf{f}\right)[\mathrm{Ni} / \mathrm{H}] ; \mathbf{g}\right)[\mathrm{Ba} / \mathrm{H}]$; and g) $[\mathrm{Eu} / \mathrm{H}]$.

note, however, that the correction factor cannot be used directly to modify the Ba abundance (and rather, the comparison of nonLTE to LTE line profiles is requisite). Consequently, in consideration of these findings as well as those of the current study, the sensitivity of the $6496 \AA$ transition to departures from LTE appears to be a minor and secondary source of error for the M 15 target stars.

\section{Chemical abundances in the M 15 sample}

The wavelength range and resolution of the spectra observed for this study allowed for the analysis of two Ca I lines, one Ni I line, one Ba II line, one La II line and two Eu II lines (see Table 2). Abundances from these lines were determined using the spectrum synthesis routine, synth, in MOOG. For each of the stars in the sample Table 11 lists the chemical abundances and the associated fitting errors, which were determined as the greatest change in abundance for possible fits to the spectral feature. Also listed are the $\mathrm{C} 09$ parameters and chemical abundances, $\xi_{\mathrm{W} 13}$ and the $\mathrm{S} / \mathrm{N}$ measured here.

To extract further information from the current M 15 sample, groups of stars were identified with similar stellar parameters and low Ba abundances. Due to the noise limitations, the key spectral features of Eu and La could not be measured confidently for these individual spectra. However they could be combined into two summed spectra such that the noise was sufficiently reduced to estimate the "summed" heavy element abundances. Table 6 gives the mean parameters for each set of spectra including the mean $[\mathrm{Ba} / \mathrm{Fe}]$ for each set, the $[\mathrm{Ba} / \mathrm{Fe}]$ derived from the summed spectra, the upper limits on $[\mathrm{Eu} / \mathrm{Fe}]$ for both summed spectra, and upper limit for $[\mathrm{La} / \mathrm{Fe}]$ for just one of the summed spectra.

Figure 5 shows the component spectra and the resulting summed spectra of A and B in the regions about the three key heavy element spectral features. The component spectra of B are much noisier than $\mathrm{A}$ as expected due to being fainter (hotter) on the RGB and due to it being an inherently weak line, the La spectral line profile was still too weak to be detected above the noise.
Table 6. Average stellar parameters of two sets of summed spectra and the resulting heavy element abundances.

\begin{tabular}{l|cc}
\hline \hline & $A$ & $B$ \\
\hline$\langle\mathrm{S} / \mathrm{N}\rangle$ & $121 \pm 25$ & $70 \pm 11$ \\
$\left\langle T_{\text {eff }}\right\rangle$ & $4949 \pm 116$ & $5216 \pm 43$ \\
$\langle\log g\rangle$ & $2.1 \pm 0.3$ & $2.6 \pm 0.1$ \\
$\langle[\mathrm{Fe} / \mathrm{H}]\rangle$ & $-2.3 \pm 0.1$ & $-2.4 \pm 0.1$ \\
$\left\langle\xi_{\mathrm{W} 13}\right\rangle$ & 1.7 & 1.6 \\
$\langle[\mathrm{Ba} / \mathrm{Fe}]\rangle$ & $-0.16 \pm 0.05$ & $-0.33 \pm 0.17$ \\
{$[\mathrm{Ba} / \mathrm{Fe}]$} & $-0.15 \pm 0.08$ & $-0.39 \pm 0.15$ \\
{$[\mathrm{Eu} / \mathrm{Fe}]$} & $<0.42$ & $<0.41$ \\
{$[\mathrm{La} / \mathrm{Fe}]$} & $<0.37$ & - \\
& & \\
$\mathrm{C} 09 \mathrm{ID}$ & $A=18815,42362,27889$ \\
& $B=35961,23216,23153,8927$ \\
\hline
\end{tabular}

Hence no upper limit on the La abundance could be extracted for summed spectrum B. The goal of the summed spectra analysis was to investigate the Eu abundance at low $\mathrm{Ba}$ abundances. This will be discussed further in the analysis of the results.

The analysis of this sample of stars in M 15 must be considered in light of the results of the previous studies. The mean values for the metallicity, $\mathrm{Ba}, \mathrm{Eu}$ and $\mathrm{La}$ abundances for each key study of M 15 are given in Table 7, where the C09 metallicity represents the mean metallicity for this sample.

What is noticeable is that the mean $\mathrm{Ba}$ abundance derived in this study is some 0.1 dex greater than for the majority of the other studies. Looking particularly at S97 and S00b, which have sizeable samples likely to sample the large range in Ba abundances, there is an offset of +0.05 dex to the mean $\mathrm{Ba}$ abundance of S97 and an offset of +0.10 to the mean Ba abundance of S00b. Both studies have dispersions in Ba abundance which are comparable to the spread in $\mathrm{Ba}$ measured for this sample. Hence the large range of Ba values for M 15 is confirmed in this study but the absolute abundances appear offset to previous studies. However it should again be noted that the 63 stars analysed here are the largest sample of M 15 stars for which Ba abundances have been determined, the next largest sample for $\mathrm{Ba}$ abundances 

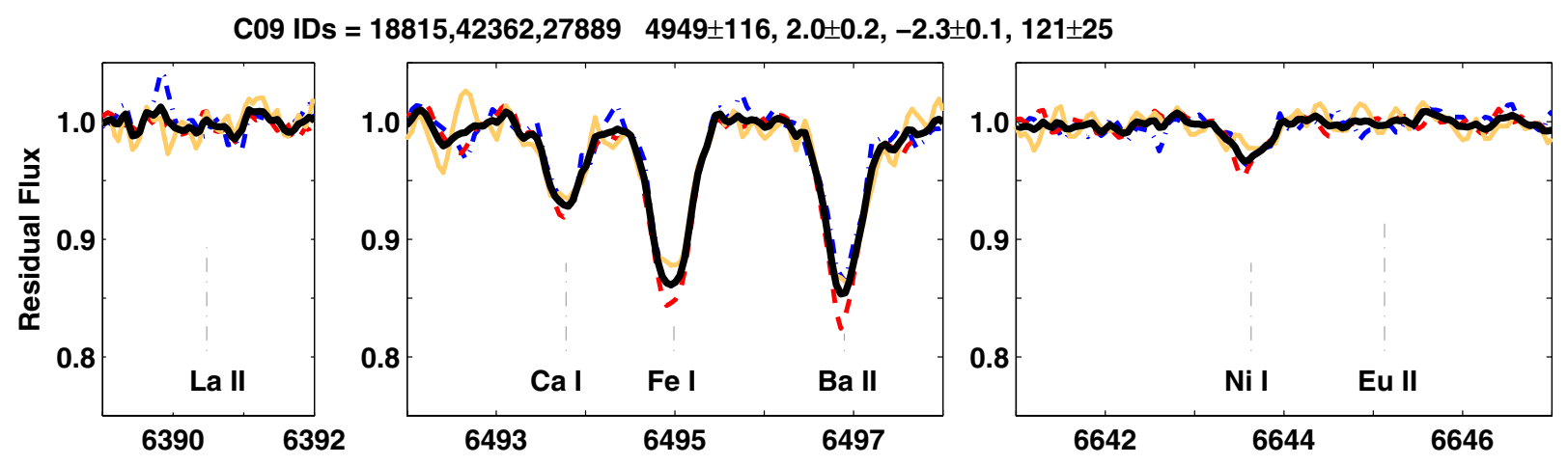

C09 IDs $=35961,23216,23153,8927 \quad 5216 \pm 43,2.6 \pm 0.1,-2.4 \pm 0.1,70 \pm 11$
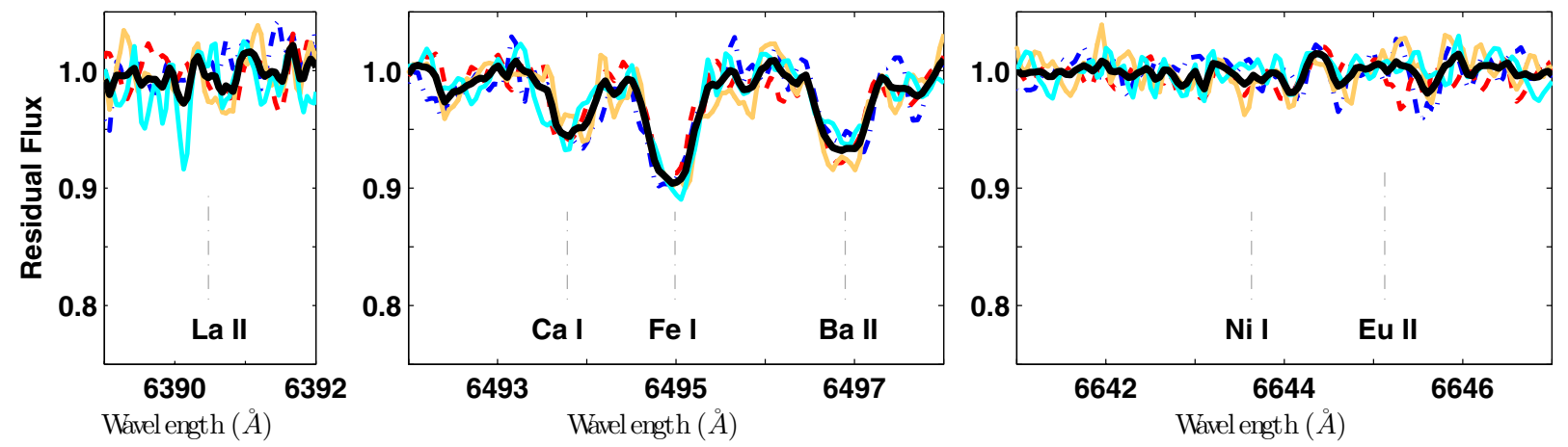

Fig. 5. Component spectra (blue, red, orange) and resulting summed spectrum (black) for A in the top panels, and the four component spectra (blue, red, orange, cyan) and resulting summed spectrum (black) for B in the bottom panels, in the regions of the La II, Ba II and Eu II spectral lines respectively. The C09 IDs for each set of spectra and the resulting mean stellar parameters $\left(T_{\text {eff }}, \log g,[\mathrm{Fe} / \mathrm{H}]\right)$ and $\mathrm{S} / \mathrm{N}$ for the final summed spectrum for each of A and B are given.

Table 7. The number of stars in the sample, the mean and standard deviations for $[\mathrm{Fe} / \mathrm{H}],[\mathrm{Ba} / \mathrm{H}],[\mathrm{La} / \mathrm{H}]$ and $[\mathrm{Eu} / \mathrm{H}]$ for each key study.

\begin{tabular}{cccccc}
\hline \hline & $N_{\text {star }}$ & $\langle[\mathrm{Fe} / \mathrm{H}]\rangle \pm \sigma$ & $\langle[\mathrm{Ba} / \mathrm{H}]\rangle \pm \sigma$ & $\langle[\mathrm{La} / \mathrm{H}]\rangle \pm \sigma$ & $\langle[\mathrm{Eu} / \mathrm{H}]\rangle \pm \sigma$ \\
\hline W13 & 63 & - & $-2.20 \pm 0.26$ & $-2.05 \pm 0.15$ & $-1.72 \pm 0.15$ \\
Sk11 & 3 & $-2.55 \pm 0.02$ & $-2.10 \pm 0.12$ & $-2.07 \pm 0.23$ & $-1.72 \pm 0.29$ \\
DO10 & 57 & - & $-2.152 \pm 0.412$ & - & - \\
C09 & 84 & $-2.341 \pm 0.007$ & - & - & - \\
O06 & 7 & $-2.40 \pm 0.05$ & $-2.56 \pm 0.18$ & $-2.46 \pm 0.25$ & $-1.85 \pm 0.24$ \\
S00b & 31 & $-2.37 \pm 0.05$ & $-2.25 \pm 0.21$ & - & - \\
S00a & 3 & $-2.28 \pm 0.05$ & $-2.28 \pm 0.19$ & - & $-1.41 \pm 0.31$ \\
S97 & 18 & $-2.40 \pm 0.04$ & $-2.30 \pm 0.21$ & - & $-1.91 \pm 0.21$ \\
\hline
\end{tabular}

Notes. ${ }^{(*)}$ RGB stars only.

being D010 with 57 stars. This sample is also complete along the RGB based on the selection criteria for C09.

The comparison to DO10 is unique in that both D010 and this study are based on slightly different subsamples of C09. Interestingly the mean $\mathrm{Ba}$ abundances of these two studies are in reasonable agreement although the spread reported in D010 is greater. As was discussed in Sect. 3, the $\xi$ values determined in $\mathrm{C} 09$ resulted in a trend of $\mathrm{Ba}$ abundance with $\xi$. Hence the mean Ba abundance from D010 must be considered in light of this, as no adjustment to the $\xi$ values was made. The equivalent width of this alternate subsample of C09 were provided (D'Orazi, priv. comm.) in order to make a better comparison of the Ba abundances using the $\log g-\xi$ relation derived here.

Considering the mean abundances for $\mathrm{La}$ and $\mathrm{Eu}$ given in Table 7, the mean La abundance agrees well with Sk11, but O06 is overestimated in comparison. On the other hand the mean $\mathrm{Eu}$ abundance for this study lies within the range of values determined for the other studies.
The offset in Ba abundance between the studies can be attributable to differences in the measurement techniques and analysis methods used to derive the stellar parameters and abundances. As a key example, the sample of 18 stars in S97 were analysed by the measurement of equivalents widths which were then used in a curve-of-growth analysis to determined the stellar parameters and abundances, whereas for C09 the stellar parameters were determined via photometry and fiducial relations configured for M 15. In particular, the Ba abundance determined in S97 was based on three Ba lines for which equivalent widths were measured. Two of the lines (6141.73 $\AA, 6496.91 \AA)$ were typically measured to have equivalent widths at $\sim 130 \mathrm{~m} \AA$, while the third (5853.69 $\AA$ ) at $\sim 80 \mathrm{~m} \AA$.

There is a similar difference in analysis method with DO10 for which equivalent width techniques were also employed in the analysis of the single Ba line at $6141.73 \AA$.

As S97 reports the equivalent widths that were measured for the 18 stars, and the equivalent widths from the DO10 analysis 

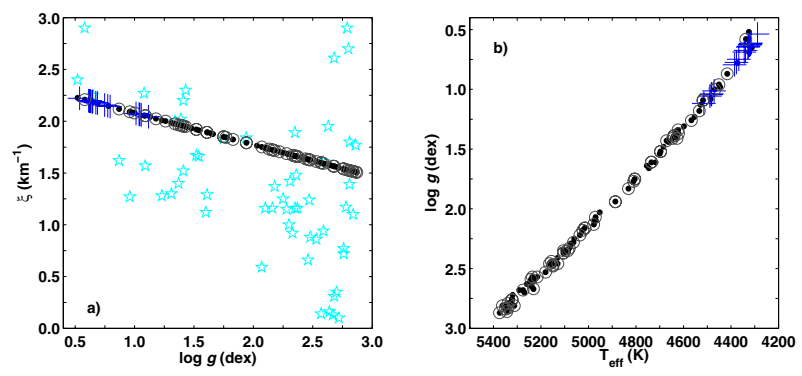

Fig. 6. C09 stellar parameters and W13 $\xi$ attributed to the S97 and DO10 samples. a) $\xi$ against $\log g$ for $\mathrm{C} 09$ (black dot), $\mathrm{S} 97_{\mathrm{W} 13}$ (blue plus) and DO10 $\mathrm{W}_{13}$ (grey circle) and DO10 (cyan star). b) HR diagram for $\mathrm{C} 09, \mathrm{~S} 97_{\mathrm{W} 13}$ and $\mathrm{DO} 10_{\mathrm{W} 13}$.

were also provided privately by the authors, we pursued extending the current analysis to include the stars from these papers.

As outlined in Table 4 six of the stars in S97 are already part of the current sample. Four of these stars are also part of the DO10 sample. In total there are 38 stars in common between DO10 and W13. Using the corresponding C09 parameters with the modified $\xi$ value, the $\mathrm{Ba}(6141.73 \AA$, $6496.91 \AA)$ and $\mathrm{Eu}(6645 \AA)$ abundances were re-determined using the S97 equivalent widths and the DO10 equivalent widths for the Ba (6141.73 ̊) abundance.

Simple low order polynomial relations were derived for $T_{\text {eff }}$ and $\log g$ as functions of $V$ based on the entire C09 sample. These functions were then used to calculate the corresponding $T_{\text {eff }}$ and $\log g$, and from $\log g$ the $\xi$, for each of the 12 S97 stars and 19 DO10 stars. The re-analysis of the DO10 and S97 samples using C09/W13 parameters and the resulting abundances are referred to as $\mathrm{S} 97_{\mathrm{W} 13}$ and $\mathrm{DO} 10_{\mathrm{W} 13}$ for the remainder of the paper.

Figure 6a shows $\xi$ compared with $\log g$ for the C09 (black dot), DO10 ${ }_{\mathrm{W} 13}$ (grey circle) and $\mathrm{S} 97_{\mathrm{W} 13}$ (blue plus). The original $\xi$ values used in DO10 (cyan star) are shown for comparison. Figure $6 \mathrm{~b}$ is the corresponding HR diagram for $\mathrm{C} 09$, DO10 ${ }_{\mathrm{W} 13}$ and $\mathrm{S} 97_{\mathrm{W} 13}$.

Using these parameters, and assuming a metallicity of $[\mathrm{Fe} / \mathrm{H}]=-2.341 \mathrm{dex}(\mathrm{C} 09)$, stellar models were interpolated using ATLAS9 as for the stellar models for the W13 sample. Then, using the equivalents widths from each study, the abundances for $\mathrm{Ba}$ and $\mathrm{Eu}$ were derived using the blends routine in MOOG taking account of hyperfine structure using the same linelists as used in the spectrum synthesis.

The cross-over stars between the three studies were investigated to look for potential systematic trends between the samples. Table 8 lists the six stars in common between W13, S97 and DO10 with their respective IDs, the Ba equivalent widths from S97 and DO10, and the resulting derived abundances where possible $\left(\mathrm{S} 97_{\mathrm{W} 13}, \mathrm{DO} 10_{\mathrm{W} 13}\right)$. While two Eu lines were used in the spectrum synthesis analysis in this paper only the abundance determined for the Eu line in common with S97 (6645 A) was used to determine the bias between the techniques.

Two effects can be seen here and are illustrated in Fig. 7, which shows the $\Delta[\mathrm{Ba} / \mathrm{H}]$ between the cross-matched stars of $\mathrm{W} 13, \mathrm{~S} 97_{\mathrm{W} 13}$ and DO10 $0_{\mathrm{W} 13}$. Although for just a sample of six stars there is a bias of $\sim 0.14$ dex between the $[\mathrm{Ba} / \mathrm{H}]$ abundances derived from the spectrum synthesis in W13 compared with the S97 $7_{\mathrm{W} 13}[\mathrm{Ba} / \mathrm{H}]$ abundances derived from the equivalent width measurements. This first effect shows that even for the same lines differences in measurements and analysis techniques lead to a difference in derived abundances.

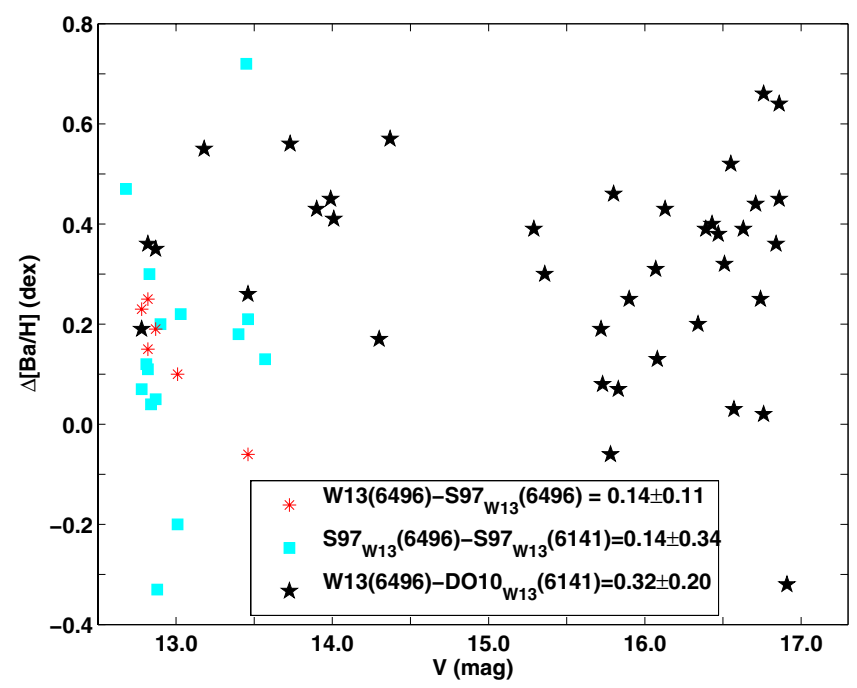

Fig. 7. Comparison of the $[\mathrm{Ba} / \mathrm{H}]$ derived for the stars in common between $\mathrm{W} 13, \mathrm{~S} 97_{\mathrm{W} 13}$ and DO10 $0_{\mathrm{W} 13}$ as per the legend against $V$ magnitude. Biases and dispersions between each sample are listed.

The second effect is illustrated by the comparison of the $\mathrm{S} 97_{\mathrm{W} 13}[\mathrm{Ba} / \mathrm{H}]$ values derived from the $6496 \AA$ line compared with the $6141 \AA$ line. There is also a bias of $\sim 0.14$ dex combined with a significant scatter $(\sim 0.34)$. Although great care was taken here deriving the $\xi$ values this discrepency between the two strong lines measured in the same study may show the derived relation is still not optimised.

Hence not only differences between measurement techniques and analysis must be taken account of, but also differences in the abundances derived by the same method but of two different $\mathrm{Ba}$ lines. This is reflected in the comparison of W13 to the DO $10_{\mathrm{W} 13}[\mathrm{Ba} / \mathrm{H}]$, which is a comparison of spectrum synthesis to equivalent width determinations as well as a comparison between the $6496 \AA$ and $6141 \AA$ lines. The bias between these two samples is $\sim 0.32 \pm 0.20$ dex, which equates to the combination of the biases produced by these two effects.

In addition, while there is no particular trend of $\Delta[\mathrm{Ba} / \mathrm{H}]$ with $V$, the brighter stars show a clear offset but the fainter stars have a much greater dispersion and less systematic offset. At the faint end the noise is greater, particularly shown by the greater fitting errors measured for W13, which can for example affect the placement of the continuum and so the derived abundance.

Also there are the issues between measurement and analysis techniques, and that it is a comparison between two different $\mathrm{Ba}$ lines. The comparison between the S97 measurements shows also a large scatter in values. As much as great care was taken in deriving the $\xi$ values this discrepency between the two strong lines measured in the same study may show the derived relation is still not optimised.

The bias for Eu calculated between $\mathrm{W} 13$ and $\mathrm{S} 97_{\mathrm{W} 13}$ is $\Delta[\mathrm{Eu} / \mathrm{H}]=0.01 \pm 0.09 \mathrm{dex}$, which is negligible. The dispersion in the bias reflects the random errors as expected. This is not unexpected as the differences in the Ba abundances are most likely driven by the microturbulence, the effects of which are negligible on the weak spectral lines of Eu. The sensitivity of strong Ba features to microturbulence makes inter-study comparisons more problematic, even after considerations of differences in measurement and analysis techniques. The spread in the values of $\Delta[\mathrm{Ba} / \mathrm{H}]$ are further evidence of the difficulty in obtaining consistency between studies. 
Table 8. Ba equivalent width (EW) measurements from $\mathrm{S} 97$ and DO10 and the corresponding $[\mathrm{Ba} / \mathrm{H}] \mathrm{and}[\mathrm{Eu} / \mathrm{H}]$ derived from these measurements based on the C09 parameters and W13 $\xi$ for the cross-over stars between W13, S97 and DO10.

\begin{tabular}{|c|c|c|c|c|c|c|c|c|c|c|}
\hline \multirow[b]{2}{*}{ C09 ID } & \multirow[b]{2}{*}{ S97 ID } & \multicolumn{3}{|c|}{$\mathrm{Ba} E W(\mathrm{m \AA})$} & \multicolumn{4}{|l|}{$[\mathrm{Ba} / \mathrm{H}]$} & \multicolumn{2}{|l|}{$[\mathrm{Eu} / \mathrm{H}]$} \\
\hline & & S97 6496 & S97 6141 & DO10 6141 & W13 6496 & S97 ${ }_{W_{13}} 6496$ & S97 ${ }_{W 13} 6141$ & DO10 $_{\mathrm{w}_{13}} 6141$ & W13 6645 & S97 $7_{\mathrm{W} 13} 6645$ \\
\hline 43788 & K825 & 136 & 137 & 137 & -2.27 & -2.52 & -2.63 & -2.63 & -1.85 & -1.84 \\
\hline 40825 & K386 & 129 & 133 & 140 & -2.42 & -2.65 & -2.72 & -2.61 & -1.94 & -1.87 \\
\hline 4099 & K341 & 146 & 146 & - & -2.27 & -2.42 & -2.53 & - & -1.67 & -1.66 \\
\hline 31914 & K757 & 151 & 147 & 154 & -2.04 & -2.23 & -2.28 & -2.39 & -1.67 & -1.72 \\
\hline 41287 & K702 & 130 & 131 & - & -2.36 & -2.46 & -2.26 & - & -1.90 & -2.07 \\
\hline 3137 & K387 & 143 & 139 & 133 & -2.02 & -1.96 & -2.17 & -2.28 & -1.73 & -1.65 \\
\hline
\end{tabular}

Notes. The W13 abundances are based on spectrum synthesis.
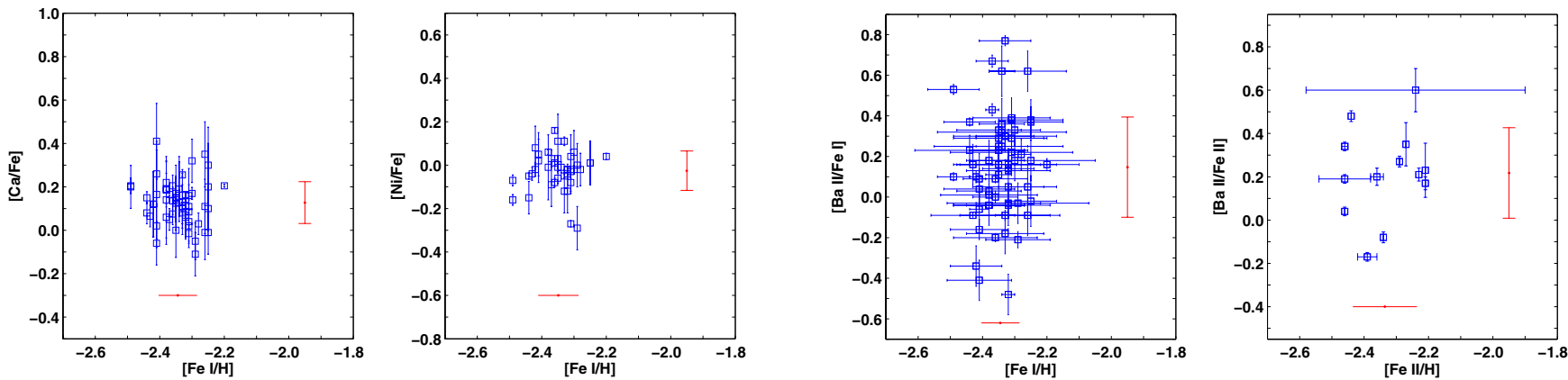

Fig. 8. Comparisons between chemical abundances measured here with $\mathrm{Fe}$ from $\mathrm{C} 09$ for this sample: a) $[\mathrm{Ca} \mathrm{I} / \mathrm{Fe}] \mathrm{vs}$. $[\mathrm{Fe} \mathrm{I} / \mathrm{H}]$; b) $[\mathrm{Ni} \mathrm{I} / \mathrm{Fe}]$ vs. $[\mathrm{Fe} \mathrm{I} / \mathrm{H}]$; c) $[\mathrm{Ba} \mathrm{II} / \mathrm{H}]$ vs. $[\mathrm{Fe} \mathrm{I} / \mathrm{H}]$; and d) $[\mathrm{Ba} \mathrm{II} / \mathrm{H}]$ against $[\mathrm{Fe} \mathrm{II} / \mathrm{H}]$. Errors for $\mathrm{Ca}$, Ni and Ba reflect the fitting errors of the spectrum synthesis. Mean values and uncertainties for each sample are shown in red.

The goal of this exploration was to see how to combine these samples. The offsets in themselves could be applied but the large scatter, particular between $\mathrm{W} 13$ and $\mathrm{DO} 10_{\mathrm{W} 13}$ for the faint stars, may reflect too many issues with the difference between the samples and techniques. It was decided that combining these samples potentially causes more problems then it solves but the samples can be analysed alongside each other for comparison as three distinct internally consistent analyses.

Table 9 lists the 12 stars from S97 and the 19 stars from DO10, the stellar parameters calibrated to C09 that were used to generate the atmospheric models and the resulting $[\mathrm{Ba} / \mathrm{H}]$ (from the $6496 \AA$ line only for S97) and [Eu/H] abundances (for S97 only).

\subsection{Abundance distributions}

Ca abundances were determined for 62 of the 63 stars while $\mathrm{Ni}$ abundances were determined for 40 of the 63 stars. From the analysis in C09 23 of the 63 stars have derived $\mathrm{O}$ abundances and 49 have derived $\mathrm{Na}$ abundances (see Table 1). Figure $8 \mathrm{a}$ and $b$ show the distribution of $[\mathrm{Ca} / \mathrm{Fe}]$ and $[\mathrm{Ni} / \mathrm{Fe}]$ respectively compared to $[\mathrm{Fe} / \mathrm{H}]$ for the M 15 sample along with the sample mean values and standard deviations in red.

Both $\mathrm{Ca}$ and Ni show a very tight distribution similar in magnitude to that of $[\mathrm{Fe} / \mathrm{H}]$, implying that there is little star-to-star variation within the cluster of the abundances of these two elements. As described in full in $\mathrm{C} 09, \mathrm{O}$ and $\mathrm{Na}$ both have a large spread of abundance values between stars in M 15. However, using the values from $\mathrm{C} 09$, no trend was found between $\mathrm{Na}$ nor $\mathrm{O}$ with any of the three heavy elements.

Figure $8 \mathrm{c}$ and $\mathrm{d}$ compares the derived $[\mathrm{Ba} / \mathrm{H}]$ values with the iron abundances determined from $\mathrm{Fe} \mathrm{I}$ and Fe II lines in C09. There is no trend of $\mathrm{Ba}$ with $\mathrm{Fe}$ for either species and the larger spread of Ba compared with $\mathrm{Fe}$ is distinct.

\subsection{Heavy elements}

The primary goal of this study was to investigate $\mathrm{Ba}$ and $\mathrm{Eu}$ abundances in a large sample of M 15 giant stars. While Ba could be measured for each of the 63 stars, Eu was measured for only 20 of the stars and La was measured for just 13 of the stars. Figure 9 compares the derived $[\mathrm{Ba} / \mathrm{H}],[\mathrm{La} / \mathrm{H}]$ and $[\mathrm{Eu} / \mathrm{H}]$ values with the stellar parameters.

As discussed in Sect. 3 the Ba II spectral feature considered here is very sensitive to $\xi$ but there is clearly no trend of $[\mathrm{Ba} / \mathrm{H}]$ with $\xi_{\mathrm{W} 13}$ despite the large spread in $[\mathrm{Ba} / \mathrm{H}]$ values. This, as well as the uniform $\mathrm{Ca}$ abundances, for which one of the lines was also sensitive to $\xi$, confirms the appropriate derivation of the $\xi-\log g$ relation carried out for this sample.

The observations suffered from greater levels of noise in the lower part of the RGB (fainter stars) hence greater uncertainties are reported. The subsample for which $\mathrm{La}$ and $\mathrm{Eu}$ abundances could be determined from their respective weak spectral features were necessarily located high on the RGB (brightest stars).

Figure 10 combines the samples from the key studies of heavy elements in M15, including the W13 sample. The S97 sample is that as reported in that paper. Despite the range in measurement types and analysis techniques there is a clear trend of $[\mathrm{Ba} / \mathrm{H}]$ with $[\mathrm{Eu} / \mathrm{H}]$, and $[\mathrm{La} / \mathrm{H}]$ with $[\mathrm{Eu} / \mathrm{H}]$ even with the dispersion between the studies. However, as discussed in Sect. 5, there are distinct offsets between the studies which in a basic comparison, such as in Fig. 10, may blur any more refined trend in the GC itsself. Hence for the rest of this paper only the reanalysis of the S97 and DO10 samples are considered and are displayed separately to the W13 sample.

Figure $11 \mathrm{a}$ and $\mathrm{b}$ compares $[\mathrm{La} / \mathrm{H}]$ with $[\mathrm{Ba} / \mathrm{H}]$ and $[\mathrm{La} / \mathrm{H}]$ with $[\mathrm{Eu} / \mathrm{H}]$ for the subsample of $13 \mathrm{~W} 13$ stars for which all three species could be measured, and Fig. 11c compares $[\mathrm{Ba} / \mathrm{H}]$ with $[\mathrm{Eu} / \mathrm{H}]$ for the 20 stars with both $\mathrm{Eu}$ and $\mathrm{Ba}$ abundances. Figure $11 \mathrm{~d}$ compares $[\mathrm{Ba} / \mathrm{H}]$ with $[\mathrm{Eu} / \mathrm{H}]$ for the 18 stars in $\mathrm{S} 97$. 

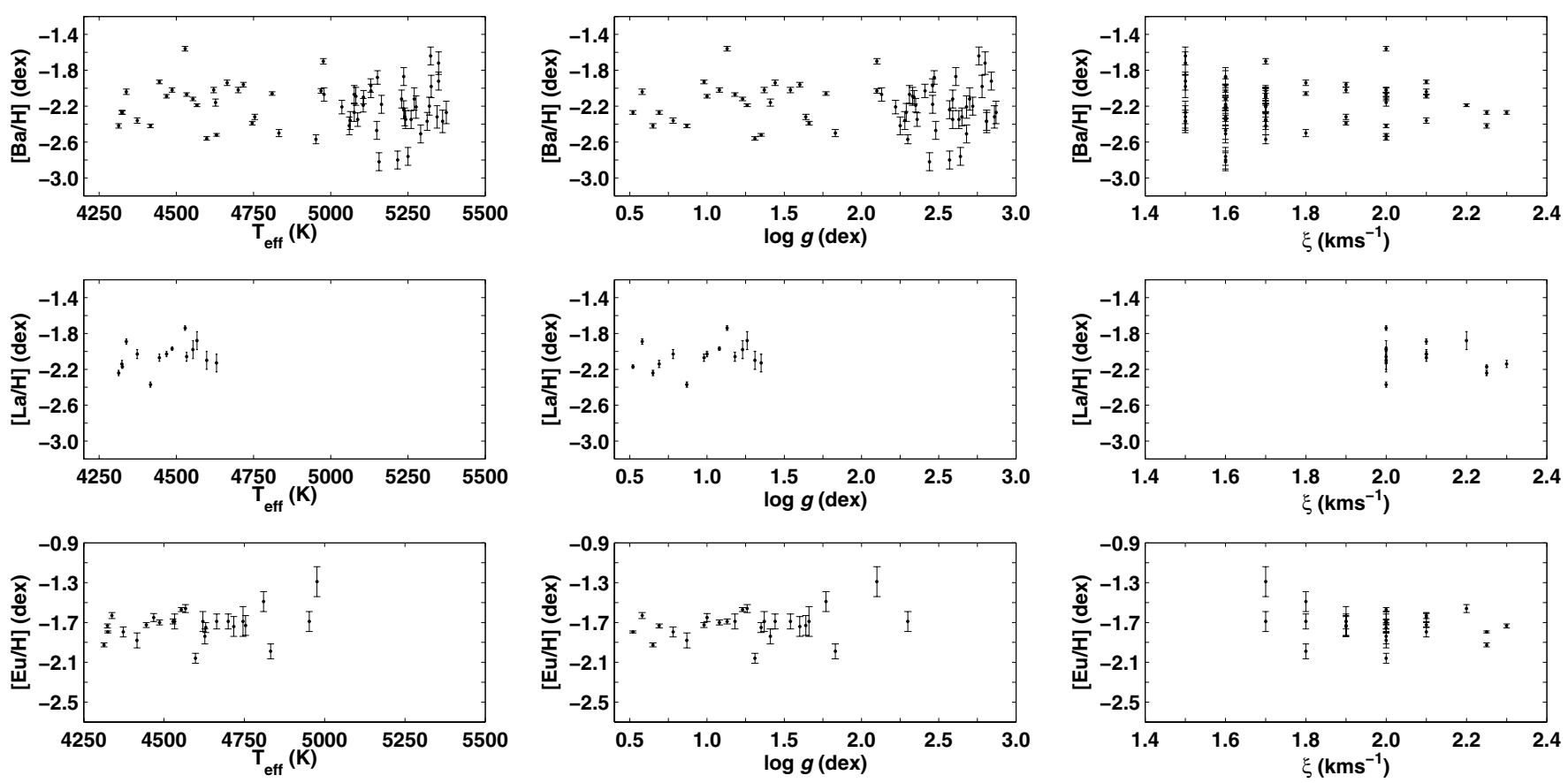

Fig. 9. Comparison of the $[\mathrm{Ba} / \mathrm{H}],[\mathrm{La} / \mathrm{H}]$ and $[\mathrm{Eu} / \mathrm{H}]$ values with the stellar parameters from $\mathrm{C} 09$. Errors on $[\mathrm{Ba} / \mathrm{H}]$ and $[\mathrm{La} / \mathrm{H}]$ are spectrum synthesis fitting errors. Errors on $[\mathrm{Eu} / \mathrm{H}]$ are the spectrum synthesis fitting errors if just one line was measured, otherwise the standard deviation of the two Eu lines summed in quadrature with the fitting error.
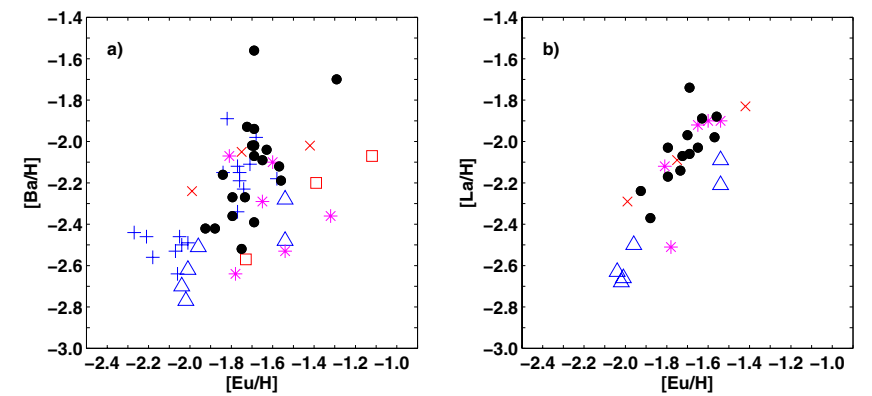

Fig. 10. a) $[\mathrm{Ba} / \mathrm{H}]$ and b) $[\mathrm{La} / \mathrm{H}]$ compared with $[\mathrm{Eu} / \mathrm{H}]$ for the $\mathrm{W} 13$ (black dot) sample compared with S97 (blue plus), S00a (red square), O06 (blue triangle), and Sk11 (RGB: red cross, RHB: pink asterix) samples where available.

Included on each graph is the line of best fit to each dataset where the weighting of each point was defined as the inverse of the errors summed in quadrature. The slope $(\mathrm{m})$, correlation coefficient $(r)$ and $p$-value $(p)$ for each comparison is given. Also included in red are upper limits in the cases for which a line could not be accurately measured but was still detectable. The limits derived from the two summed spectra are also included in grey.

For each comparison of the W13 samples the calculated p-value is less than 0.05 which indicates that the linear relation of these data is unlikely to have been produced by chance and is therefore significant in each case (i.e. the null hypothesis, that there is no correlation, is rejected). However the $\mathrm{S} 97_{\mathrm{W} 13}$ sample fails this test. The strongest correlation is between $\mathrm{La}$ and $\mathrm{Ba}$, with the La to Eu correlation being of a similar magnitude and both with values of the correlation co-efficient (r) being greater than 0.7 , which indicates a strong positive linear relationship.

For W13 the correlation between $\mathrm{Ba}$ and $\mathrm{Eu}$ is also significant based on the $\mathrm{p}$-value, but the value of $\mathrm{r}$ indicates only a moderate positive linear relationship. The graph itsself shows a great deal of dispersion with at least three extrema, unlike the comparably clean (albeit smaller sample) relation of $\mathrm{La}$ to $\mathrm{Eu}$. There is some dispersion in the $\mathrm{La}$ and $\mathrm{Ba}$ relation. The correlations of $\mathrm{La}$ to Eu have been confirmed for three other GCs, as well as M 15, in the investigation of literature sources in Roederer (2011).

However for both samples, there does appear to be evidence of a bimodal distribution in $[\mathrm{Ba} / \mathrm{H}]$ as seen in Fig. 11c and $\mathrm{d}$. For $\mathrm{W} 13$, at $[\mathrm{Ba} / \mathrm{H}] \sim-2.20$ there is a clear separation in the distribution, with the low $\mathrm{Ba}$ abundance group being more dispersed in both $\mathrm{Ba}$ and $\mathrm{Eu}$ than the high $\mathrm{Ba}$ abundance group, which appears to have a much tighter distribution (ignoring the high $\mathrm{Ba}$ extrema). For $\mathrm{S} 97_{\mathrm{W} 13}$ the separation occurs at $[\mathrm{Ba} / \mathrm{H}] \sim$ -2.30 with the low $\mathrm{Ba}$ abundance group showing a more scatter though uniform distribution while the high $\mathrm{Ba}$ abundance group has a tight core and is more dispersed in extrema. But the W13 and $\mathrm{S} 97_{\mathrm{W} 13}$ samples are in reasonable agreement, if globally offset, in the two groupings so as to emphasise rather than diminish the separation in $[\mathrm{Ba} / \mathrm{H}]$.

In Fig. 11c the upper limits on the determination of Eu for 6 noisy W13 spectra are shown in red and also fall so as to agree with the bimodal distribution. The upper limits on the two sets of summed spectra are also shown in grey. The two sets of spectra were selected as having components of low $\mathrm{Ba}$ abundances in order to explore the low Eu regime, the key line of which was not resolved in the majority of the individual W13 spectra. One set (with higher $\mathrm{S} / \mathrm{N}$ ) clearly falls within the low Ba abundance mode. The second set, comprised of much noisier spectra, lies well below this mode and given how noisy the spectra are should probably be discounted.

\subsection{Bimodal Ba distribution in M 15?}

The existence of a bimodal distribution in $\mathrm{Ba}$ and $\mathrm{Eu}$ in $\mathrm{M} 15$ was raised in Sneden et al. (1997). A re-analysis of key sets of stars from Sneden et al. (1997, 2000a), Preston et al. (2006) and Otsuki et al. (2006) in the study of Sobeck et al. (2011) determined that such a bimodal distribution is not evident but 

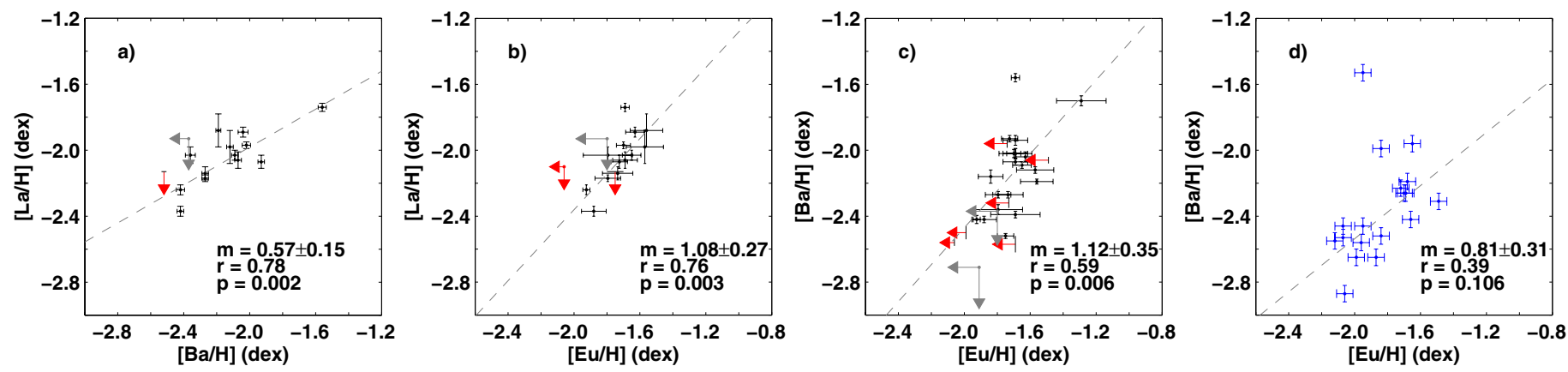

Fig. 11. Comparisons of $[\mathrm{La} / \mathrm{H}]$ with $[\mathrm{Ba} / \mathrm{H}]$ and $[\mathrm{La} / \mathrm{H}]$ with $[\mathrm{Eu} / \mathrm{H}]$ for the $13 \mathrm{~W} 13$ stars with $\mathrm{La}$, Eu and $\mathrm{Ba}$ measurements $(\mathbf{a})$ and $\mathbf{b})$ ), and $[\mathrm{Ba} / \mathrm{H}]$ with $[\mathrm{Eu} / \mathrm{H}]$ for the $20 \mathrm{~W} 13$ stars with $\mathrm{Ba}$ and Eu measurements $\mathbf{c})$. The errorbars for La and $\mathrm{Ba}$ are the fitting errors, while for Eu the errorbars are the standard deviation if two lines were measured, otherwise the errorbars are the fitting errors. The line of best fit (grey dash) and its slope (m), as well as the correlation coefficient (r) and p-value (p) of each dataset is given. Red arrows are upper limits on the abundances for particular stars. Grey arrows are limits determined for the two sets of summed spectra. d) $[\mathrm{Ba} / \mathrm{H}] \mathrm{vs}$. $[\mathrm{Eu} / \mathrm{H}]$ for the $18 \mathrm{~S} 97_{\mathrm{W} 13}$ stars.

Table 9. $[\mathrm{Ba} / \mathrm{H}]$ and $[\mathrm{Eu} / \mathrm{H}]$ derived for the 12 stars of $\mathrm{S} 97$ and the 19 stars of DO10 that are not part of the W13 sample.

\begin{tabular}{lccccccc}
\hline \hline S97 ID & $V$ & $T_{\text {eff }}$ & $\log g$ & {$[\mathrm{M} / \mathrm{H}]$} & $\xi$ & {$[\mathrm{Ba} / \mathrm{H}]$} & {$[\mathrm{Eu} / \mathrm{H}]$} \\
\hline K479 & 12.68 & 4288 & 0.54 & -2.341 & 2.2 & -1.99 & -1.84 \\
K634 & 12.81 & 4321 & 0.63 & -2.341 & 2.2 & -2.56 & -1.96 \\
K583 & 12.83 & 4327 & 0.64 & -2.341 & 2.2 & -2.55 & -2.12 \\
K490 & 12.84 & 4329 & 0.65 & -2.341 & 2.2 & -2.87 & -2.06 \\
K853 & 12.88 & 4339 & 0.67 & -2.341 & 2.2 & -2.65 & -1.99 \\
K462 & 12.90 & 4344 & 0.69 & -2.341 & 2.2 & -2.31 & -1.49 \\
K431 & 13.03 & 4377 & 0.77 & -2.341 & 2.1 & -2.46 & -1.95 \\
K144 & 13.06 & 4385 & 0.79 & -2.341 & 2.1 & -2.26 & -1.70 \\
K1040 & 13.40 & 4471 & 1.01 & -2.341 & 2.1 & -2.19 & -1.68 \\
K969 & 13.45 & 4484 & 1.04 & -2.341 & 2.1 & -1.53 & -1.95 \\
K169 & 13.47 & 4489 & 1.06 & -2.341 & 2.1 & -2.26 & -1.69 \\
K146 & 13.57 & 4514 & 1.12 & -2.341 & 2.0 & -2.53 & -2.07 \\
& & & & & & & \\
C09 ID & & & & & & & \\
\hline 34332 & 13.32 & 4451 & 0.96 & -2.41 & 2.1 & -2.42 & - \\
14574 & 13.58 & 4518 & 1.09 & -2.34 & 2.1 & -2.65 & - \\
25247 & 14.00 & 4623 & 1.34 & -2.34 & 2.0 & -2.35 & - \\
34561 & 14.07 & 4641 & 1.39 & -2.27 & 2.0 & -2.15 & - \\
29401 & 14.09 & 4646 & 1.41 & -2.34 & 2.0 & -2.78 & - \\
37415 & 14.19 & 4671 & 1.43 & -2.28 & 1.9 & -2.17 & - \\
9753 & 14.29 & 4697 & 1.52 & -2.40 & 1.9 & -2.73 & - \\
18913 & 14.44 & 4735 & 1.61 & -2.46 & 1.9 & -3.06 & - \\
31313 & 14.72 & 4805 & 1.75 & -2.28 & 1.8 & -2.08 & - \\
33507 & 15.04 & 4887 & 1.94 & -2.33 & 1.8 & -2.21 & - \\
41437 & 15.37 & 4971 & 2.07 & -2.29 & 1.8 & -2.68 & - \\
31956 & 15.54 & 5015 & 2.16 & -2.34 & 1.7 & -2.35 & - \\
30535 & 15.58 & 5024 & 2.18 & -2.24 & 1.7 & -2.07 & - \\
41279 & 15.82 & 5087 & 2.35 & -2.33 & 1.7 & -2.15 & - \\
28350 & 15.90 & 5105 & 2.37 & -2.37 & 1.7 & -2.86 & - \\
38382 & 16.19 & 5180 & 2.53 & -2.20 & 1.6 & -2.20 & - \\
24706 & 16.40 & 5232 & 2.67 & -2.42 & 1.6 & -3.08 & - \\
36971 & 16.75 & 5321 & 2.76 & -2.26 & 1.5 & -2.39 & - \\
20994 & 16.82 & 5338 & 2.79 & -2.28 & 1.5 & -2.51 & - \\
\hline & & & & & & & \\
\hline
\end{tabular}

Notes. For $\mathrm{S} 97_{\mathrm{W} 13}$ only $\lambda(\mathrm{Ba})=6496 \AA$ and $\lambda(\mathrm{Eu})=6645 \AA$ are used The C09 stellar atmosphere parameters (with $[\mathrm{M} / \mathrm{H}]=-2.341 \mathrm{dex}$ for $\left.\mathrm{S} 97_{\mathrm{W} 13}\right)$ and $\xi_{\mathrm{W} 13}$ are listed.

rather that there was a continuum of increasing $\mathrm{Ba}$ with increasing Eu.

To investigate this further Fig. 12 shows a series of histograms of $[\mathrm{Ba} / \mathrm{H}]$ for the $\mathrm{W} 13$ and $\mathrm{DO} 10_{\mathrm{W} 13}$ samples. Figure $12 \mathrm{a}$ is the histogram of the W13 sample with a binsize of 0.1 dex (blue). This is the largest M 15 sample of giant stars to-date that have been analysed for their Ba abundances, which also well samples along the RGB. A bimodal distribution is clearly seen. The histogram of the subset of bright stars from $\mathrm{W} 13(V<15.2)$ is also shown and the bimodal distribution is still clear. These stars are singled out as having the lowest associated error and hence most accurate abundance determination.

Figure $12 \mathrm{c}$ replicates Fig. 12a but for the DO10 ${ }_{\mathrm{W} 13}$ sample. This similarly large sample of 57 giant stars also well samples along the giant branch of M 15. There is no clear bimodal distribution but also no clear single Gaussian distribution either. The distribution appears rectangular in that all the bins, including the extrema, are similarly populated. That the extrema bins are thus populated argues against this distribution being a simple Gaussian. Even the bright subsample for $\mathrm{DO} 10_{\mathrm{W} 13}$ does not clarify the distribution. It is noted that the range of values is offset to W13 to more Ba-poor values as expected.

Figure $12 \mathrm{c}$ combines $\mathrm{W} 13$ with the further 12 stars from $\mathrm{S} 97_{\mathrm{W} 13}$ and the 19 stars $\mathrm{DO} 10_{\mathrm{W} 13}$ by applying the offsets specified above which corrected the $\mathrm{S} 97_{\mathrm{W} 13}$ stars and the DO10 ${ }_{\mathrm{W} 13}$ stars to the W13 system. The overall distribution is bimodal but the extrema bins are also well populated. Hence the characteristics of the W13 (bimodal) and DO10 (well-populated extrema bins) are clear and do not erase the effects of each other. However as the crossover samples have a noticable scatter between the $\mathrm{Ba}$ results, which implies that per star the $\mathrm{Ba}$ abundances from each line and study are not in good agreement, then combining the three does not produce a sample that can be considered to be internally consistent (see Sect. 5).

The threshold of the bimodal distribution in the W13 sample lies at $[\mathrm{Ba} / \mathrm{H}] \sim-2.20$ dex separating the sample into two modes, Mode I being the low $\mathrm{Ba}$ abundance mode and Mode II being the high $\mathrm{Ba}$ abundance mode. Table 10 lists the mean and standard deviation for the three samples of W13, DO10 ${ }_{\mathrm{W} 13}$ and $\mathrm{S} 97_{\mathrm{W} 13}$. Statistics are also given for the two modes for W13, DO10 ${ }_{\mathrm{W} 13}$ (assuming a threshold for DO10 $\mathrm{W} 13_{3}$ of $[\mathrm{Ba} / \mathrm{H}] \sim-2.47 \mathrm{dex}$ ) and S97 ${ }_{\mathrm{W} 13}$ (assuming a threshold of $[\mathrm{Ba} / \mathrm{H}] \sim-2.36 \mathrm{dex}$ ).

The $[\mathrm{Fe} / \mathrm{H}]$ values stay relatively constant across all means and all samples with very low associated spread reflecting the tight spread obtained in $\mathrm{C} 09$. While the absolute mean values for $\mathrm{Ba}$ are offset from each other between the three studies all show the expected large spread in values. When considering the modes the spread of the $[\mathrm{Ba} / \mathrm{H}]$ values are on a similar magnitude for each of the samples.

Regarding Eu, W13 and S97 $7_{\mathrm{W} 13}$ show good agreement in the mean for each whole sample and for the mean of Mode I. Mode II, the high $\mathrm{Ba}$ mode, shows enhanced $\mathrm{Eu}$ for $\mathrm{S} 97_{\mathrm{W} 13}$ 

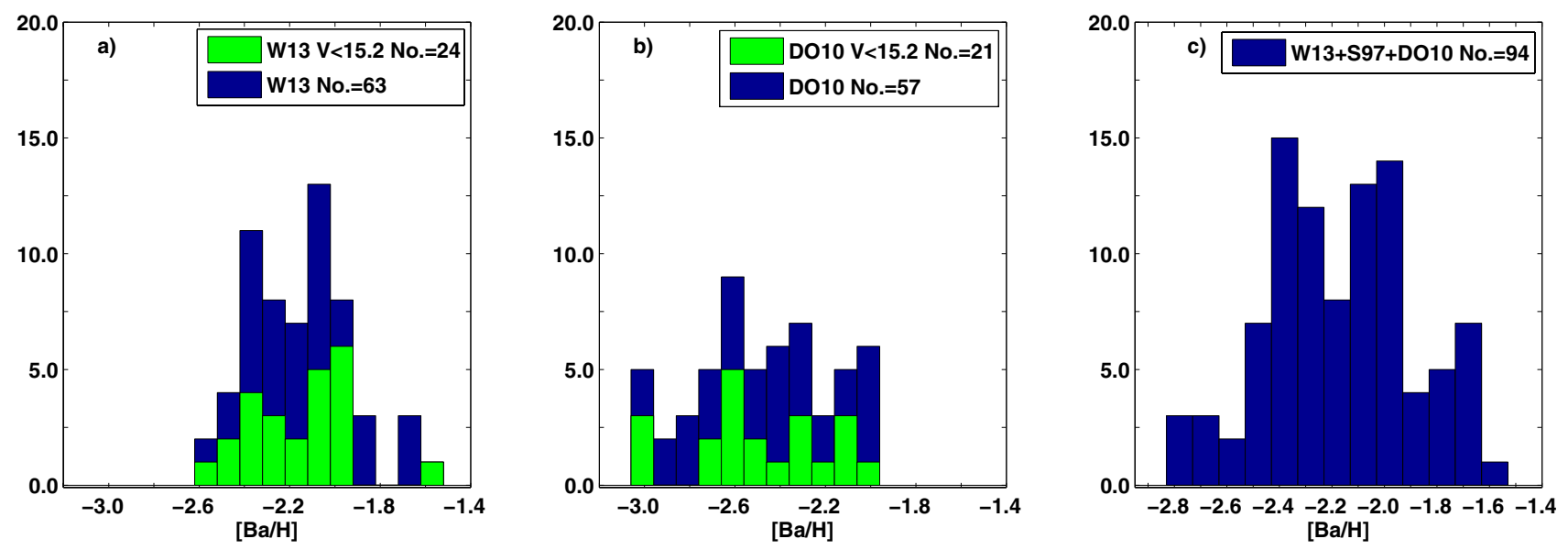

Fig. 12. Histograms exploring the three key samples of $\mathrm{M} 15$ giants in $[\mathrm{Ba} / \mathrm{H}]$ : a) all $63 \mathrm{~W} 13$ stars (blue) and 24 bright W13 stars (green); b) all $57 \mathrm{DO}_{10} \mathrm{~W} 13_{3}$ stars (blue) and 21 bright DO10 $\mathrm{W} 13_{3}$ stars (green); and c) the W13 sample combined with the further $12 \mathrm{~S} 97_{\mathrm{W} 13}$ stars and the further 19 DO10 $10_{13}$ stars with offsets applied.

Table 10. Mean and standard deviations of $[\mathrm{Fe} / \mathrm{H}],[\mathrm{Ba} / \mathrm{H}],[\mathrm{Eu} / \mathrm{H}]$, and $[\mathrm{La} / \mathrm{H}]$ derived (where possible) for the three samples of W13, DO10 1313 and $\mathrm{S} 97_{\mathrm{W} 13}$, for each whole sample and separated into the respective low (I) and high (II) Ba modes.

\begin{tabular}{|c|c|c|c|c|c|c|c|c|c|c|}
\hline & $N$ & {$[\mathrm{Fe} / \mathrm{H}]$} & $N_{\mathrm{Ba}}$ & {$[\mathrm{Ba} / \mathrm{H}]$} & $N_{\mathrm{Eu}}$ & {$[\mathrm{Eu} / \mathrm{H}]$} & $N_{\mathrm{La}}$ & {$[\mathrm{La} / \mathrm{H}]$} & {$[\mathrm{Ba} / \mathrm{Eu}]$} & {$[\mathrm{La} / \mathrm{Eu}]$} \\
\hline W13 & 63 & $-2.34 \pm 0.06$ & 63 & $-2.20 \pm 0.26$ & 20 & $-1.70 \pm 0.13$ & 13 & $-2.04 \pm 0.16$ & $-0.50 \pm 0.29$ & $-0.24 \pm 0.21$ \\
\hline $\mathrm{DO} 10_{\mathrm{W} 13}$ & 57 & $-2.33 \pm 0.06$ & 57 & $-2.47 \pm 0.31$ & - & - & - & - & - & - \\
\hline $\mathrm{S} 97_{\mathrm{W} 13}$ & 18 & $-2.33 \pm 0.03$ & 18 & $-2.36 \pm 0.28$ & 18 & $-1.78 \pm 0.17$ & - & - & $-0.58 \pm 0.33$ & - \\
\hline W13 Mode I & {$[\mathrm{Ba} / \mathrm{H}]<-2.20$} & $-2.36 \pm 0.06$ & 30 & $-2.41 \pm 0.16$ & 7 & $-1.80 \pm 0.08$ & 5 & $-2.19 \pm 0.13$ & $-0.61 \pm 0.18$ & $-0.39 \pm 0.15$ \\
\hline W13 Mode II & {$[\mathrm{Ba} / \mathrm{H}] \geq-2.20$} & $-2.33 \pm 0.05$ & 33 & $-2.00 \pm 0.16$ & 13 & $-1.65 \pm 0.13$ & 8 & $-1.95 \pm 0.11$ & $-0.35 \pm 0.21$ & $-0.30 \pm 0.17$ \\
\hline DO10 ${ }_{\mathrm{W} 13}$ Mode I & {$[\mathrm{Ba} / \mathrm{H}]<-2.47$} & $-2.33 \pm 0.05$ & 29 & $-2.74 \pm 0.18$ & - & - & - & - & - & - \\
\hline DO10 ${ }_{\mathrm{W} 13}$ Mode II & {$[\mathrm{Ba} / \mathrm{H}] \geq-2.47$} & $-2.34 \pm 0.07$ & 28 & $-2.22 \pm 0.16$ & - & - & - & - & - & - \\
\hline S97 ${ }_{\mathrm{W} 13}$ Mode I & {$[\mathrm{Ba} / \mathrm{H}]<-2.36$} & $-2.34 \pm 0.01$ & 10 & $-2.56 \pm 0.12$ & 10 & $-1.92 \pm 0.15$ & - & - & $-0.64 \pm 0.19$ & - \\
\hline S97 1 13 Mode II & {$[\mathrm{Ba} / \mathrm{H}] \geq-2.36$} & $-2.33 \pm 0.05$ & 8 & $-2.11 \pm 0.19$ & 8 & $-1.76 \pm 0.18$ & - & - & $-0.35 \pm 0.26$ & - \\
\hline
\end{tabular}

Notes. The number of stars $(N)$ use to calculate the element abundance.

compared to the mean of Mode II for W13. The spread in Eu values within each mode is of similar magnitude and not significantly smaller than that of the spread in the Ba values. While La abundances are only available for W13 the spread on the whole sample and for each mode are again similar in magnitude to the corresponding spread in $\mathrm{Eu}$.

Certainly for each sample and each element, there is a clear separation in mean values between the modes. Even for Eu and La the separation between the means of Mode I and Mode II are outside the $1 \sigma$ limit.

\section{4. s-process residuals: [Ba/Eu], [La/Eu]}

The combination of the various studies confirmed the correlation of $[\mathrm{Ba} / \mathrm{H}]$ with $[\mathrm{Eu} / \mathrm{H}]$, and $[\mathrm{La} / \mathrm{H}]$ with $[\mathrm{Eu} / \mathrm{H}]$ and the analysis here has confirmed a bimodal distribution in $\mathrm{Ba}$ and $\mathrm{Eu}$ and possibly also in La. To further explore these heavy element abundances in M 15 Fig. 13 compares $[\mathrm{Ba} / \mathrm{Eu}]$ and $[\mathrm{La} / \mathrm{Eu}]$ to $[\mathrm{Eu} / \mathrm{H}]$, $[\mathrm{Ba} / \mathrm{H}]$ and $[\mathrm{La} / \mathrm{H}]$ for both the $\mathrm{W} 13$ (black) and $\mathrm{S} 97_{\mathrm{W} 13}$ (blue) samples.

The ratios of $[\mathrm{Ba} / \mathrm{Eu}]$ and $[\mathrm{La} / \mathrm{Eu}]$ can be considered as the contribution of the $s$-process to the heavy element abundances once the contribution from the $r$-process $(\mathrm{Eu})$ has been removed. For W13 in Figs. $13 \mathrm{~b}$ and $\mathrm{f}$ there is no trend of either
$[\mathrm{Ba} / \mathrm{Eu}]$ nor $[\mathrm{La} / \mathrm{Eu}]$ with $[\mathrm{Eu} / \mathrm{H}]$. This is not unexpected, as if all the contribution from the $r$-process has been removed then the $s$-process contribution would not trend with increasing $r$ process abundances. This is borne out by the distribution lying closer to the estimated solar $r$-process only yield abundance (indicated as red dashed lines, Simmerer et al. 2004) than to the solar abundance (red dotted lines), which is a combination of both $r$ - and $s$-process contributions. However the distribution does not lie exactly on the estimated solar $r$-process only yield implying that there is some contribution of the heavy elements from some other process. This is replicated in Fig. 13a for the S97 W13 sample.

Figure $13 \mathrm{c}$ is particularly interesting as it shows whether $[\mathrm{Ba} / \mathrm{Eu}]$ shows any trend with increasing $[\mathrm{Ba} / \mathrm{H}]$, thus $s$-process contribution increasing with $s$-process abundance. The bimodal distribution in $\mathrm{Ba}$ can be seen as a plateau for Mode I, implying that this mode has had no further heavy element contribution from the $s$-process, but a distinct linear trend of increasing $[\mathrm{Ba} / \mathrm{Eu}]$ with $[\mathrm{Ba} / \mathrm{H}]$ for Mode II. Both of these distributions for each mode are replicated in the $\mathrm{S} 97_{\mathrm{W} 13}$, with the linear trend in Mode II appearing to extend to the outliers of each sample. This would imply that the stars in Mode I have undergone further Ba enhancement processes (i.e. the $s$-process).

Is this linear relation in Mode II real? As $\mathrm{La}$ and $\mathrm{Ba}$ are both $s$-process elements then an interchange of these parameters 

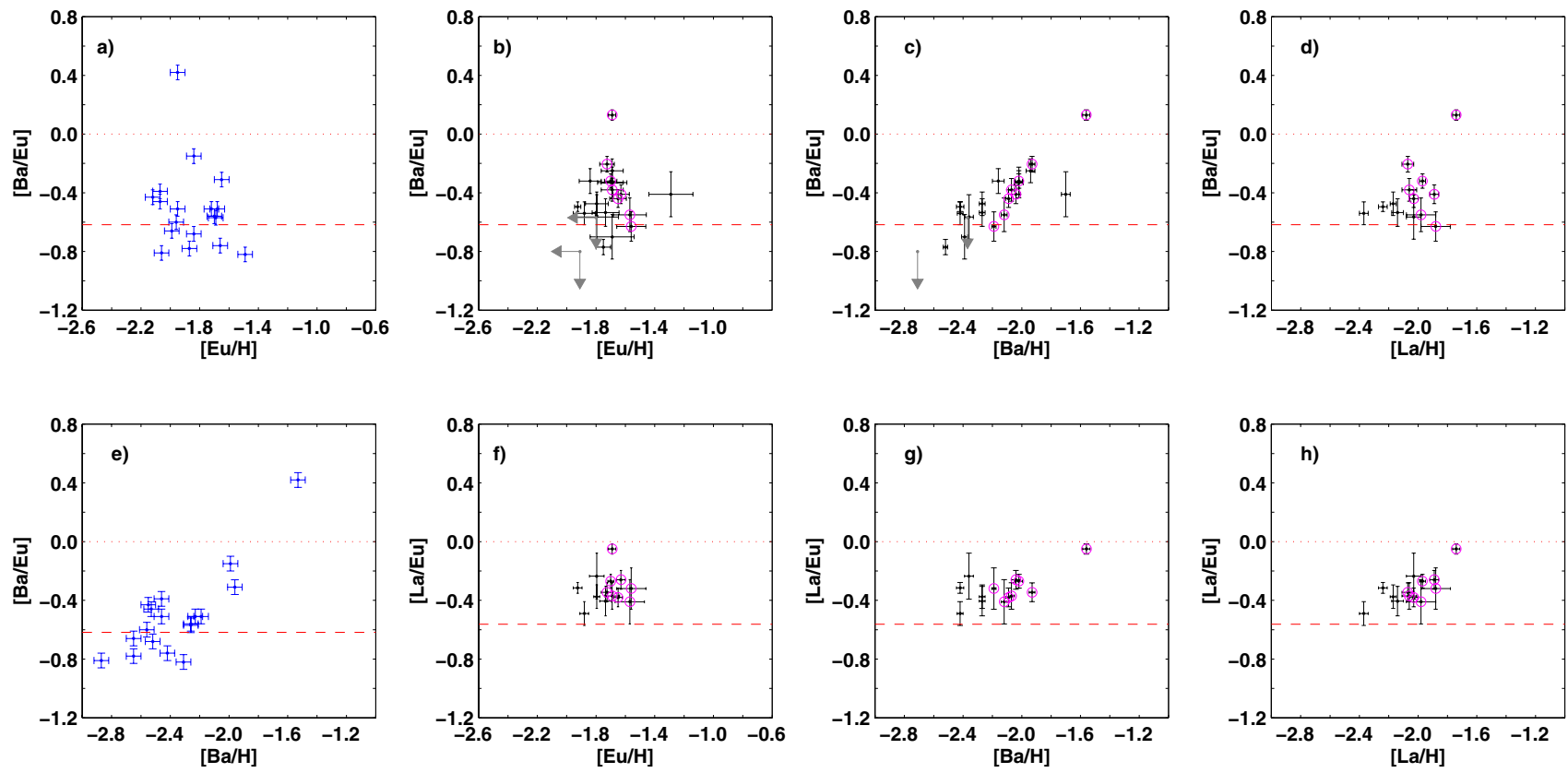

Fig. 13. a) $[\mathrm{Ba} / \mathrm{Eu}]$ against $[\mathrm{Eu} / \mathrm{H}]$ for $\mathrm{S} 97_{\mathrm{W} 13}$ (blue). Dotted red line indicates the solar abundance. Dashed red line indicates the expected abundance from $r$-process only yields (Simmerer et al. 2004). b) $[\mathrm{Ba} / \mathrm{Eu}]$ against $[\mathrm{Eu} / \mathrm{H}]$ for the W13 sample (black). Grey arrows are the upper limits derived from the set of summed spectra. Magenta circles identify those points with $[\mathrm{Ba} / \mathrm{H}]$ greater than -2.20 dex and that have measurements for $\mathrm{Ba}, \mathrm{La}$ and $\mathrm{Eu}$. c) As for b) but for $[\mathrm{Ba} / \mathrm{Eu}]$ against $[\mathrm{Ba} / \mathrm{H}]$. d) as for b) but for $[\mathrm{Ba} / \mathrm{Eu}]$ against $[\mathrm{La} / \mathrm{H}]$. e) As for a) but for $[\mathrm{Ba} / \mathrm{Eu}]$ against $[\mathrm{Ba} / \mathrm{H}]$. f) As for $\mathbf{b})$ but for $[\mathrm{La} / \mathrm{Eu}]$ against $[\mathrm{Eu} / \mathrm{H}]$. g) as for $\mathbf{b}$ ) but for $[\mathrm{La} / \mathrm{Eu}]$ against $[\mathrm{Ba} / \mathrm{H}] . \mathbf{h})$ as for $\mathbf{b})$ but for $[\mathrm{La} / \mathrm{Eu}]$ against $[\mathrm{La} / \mathrm{H}]$.

should show the same linear relation. However, as La could only be measured for 13 of the W13 sample and was not available as an equivalent width measurement in S97, then the sample for comparison is significantly reduced. To aid the investigation the eight stars with all three $\mathrm{Ba}$, La and Eu abundances that lie within Mode II are identified in all the W13 graphs of Fig. 13 as magenta circles. For Figs. 13d there is no convincing linear trend, despite the one outlier. For Figs. 13g and h, if one excludes the high La outlier, then there is no strong argument for a linear relation.

An examination of the parameters of the stars within Mode II showed no obvious trend of $\mathrm{Ba}$ abundance with $\xi$. However for five of these objects there is a distinct linear trend with $T_{\text {eff }}$ and $\log g$. Given the almost linear relations between $V$ and these two parameters, and the linear relation developed here between $\xi$ and $\log g$ it is likely that these are the cause of the observed linear trend of $[\mathrm{Ba} / \mathrm{Eu}]$ with $[\mathrm{Ba} / \mathrm{H}]$. The error analysis shows the sensitivity of the analysed $\mathrm{Ba}$ line to these parameters, which is accentuated for stronger/more enhanced features. Hence this is the most likely reason why this relation is observed in the high Ba Mode II, not the low Ba Mode I.

The La line is much weaker and hence not so sensitive to $\xi$. While there are fewer points, the lack of a linear trend should be taken as confirmation that the linear trend in $\mathrm{Ba}$ is not real. However, the location of the $[\mathrm{La} / \mathrm{Eu}]$ distribution with respect to the $r$-process only yields and the solar abundance does not exclude some contribution from the $s$-process or some other process that produces heavy elements.

\section{Heavy and light element contributions within M 15}

The existence of two modes in Ba and Eu abundances in M 15 was first proposed in S97. For both modes the mean $[\mathrm{Ba} / \mathrm{Eu}]$ determined in S97 were found to be almost exactly the same
$(\langle[\mathrm{Ba} / \mathrm{Eu}]\rangle \sim-0.40 \pm 0.05 \mathrm{dex})$ and indicative of a pollution history dominated by contributions from the $r$-process. In this study there is a separation in the mean $[\mathrm{Ba} / \mathrm{Eu}]$ values of $\sim 0.3$ dex outside the uncertainties (see Table 10), with the value for Mode I being less than that for Mode II. In the $\mathrm{S} 97_{\mathrm{W} 13}$ sample similar values to W13 were found and so a similar difference outside the uncertainties. But both indicate no extra contribution from the $s$-process, therefore this study agrees with S97 that both modes have an enrichment history dominated by the $r$-process but that Mode I has been less enriched in the heavy elements than Mode II.

However when considering the $s$-process residuals as represented by $[\mathrm{La} / \mathrm{Eu}]$ both modes have values in close agreement and well within the uncertainties, with Mode I being slightly less enhanced. Given the greater consistency that can be obtained from La measurements this can be well relied upon, but still supports the above conclusion that both Modes are $r$-process dominated with a possible greater enrichment in Mode II.

S00b confirmed the conclusions of S97 on a larger sample of M 15 stars for which $\mathrm{Ba}$ only was determined of the heavy elements. S00a investigated in detail several heavy element abundances for three of the S97 giants, two that lie in the proposed Mode I and one in Mode II. The signatures that they found were confirmation of $r$-process dominated abundances.

The heavy elements can be paired with light element counterparts based on their common sites of nucleosynthesis in order to constrain the exact nature of the source of these chemical abundance patterns. In particular, the $r$-process and $\alpha$-capture processes (producing $\mathrm{Ca}$ ) can both occur in Type II supernova. Similarly proton-capture processes (producing $\mathrm{Na}$ ) occur during the evolution up the AGB, as does the $s$-process in the thermally pulsing stage.

S97 determined that neither the $\mathrm{Ba}$ nor Eu abundances correlated with the $\alpha$-element abundances. In this study no correlation was found between $\mathrm{Ba}$ and $\mathrm{Ca}$, nor between $\mathrm{Eu}$ 

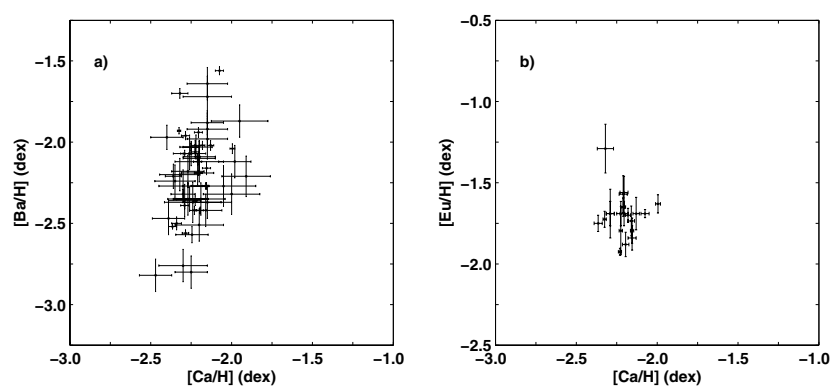

Fig. 14. Comparison of light with heavy element abundances for the W13 sample: a) $[\mathrm{Ba} / \mathrm{H}]$ against $[\mathrm{Ca} / \mathrm{H}]$; b) $[\mathrm{Eu} / \mathrm{H}]$ against $[\mathrm{Ca} / \mathrm{H}]$.

and $\mathrm{Ca}$ supporting the result found in S97 as shown in Figs. 14a and b. The conclusion drawn in S97, that is supported here, was that the mass of the star resulting in the Type II supernova that produced the $r$-process elements was insufficient to simultaneously produce the $\alpha$-elements. The $\mathrm{Ba}$ and Eu abundances derived here fall within the range of values of collated data and model yields in Chen et al. (2006). Based on this the two modes of M 15 are both indicative of pollution by Type II supernova. As concluded in Chen et al. (2006) and references therein, Type II supernova associated with stars of progenitor mass between $20 M_{\odot}$ and $40 M_{\odot}$ are the main source of $r$-process elements in the early galaxy.

With regards to the $s$-process and proton-capture elements, $\mathrm{Na}$ was measured for S97, S00b, O06 and Sk11 but no correlation between $\mathrm{Na}$ and either $\mathrm{Ba}$ or La was found. Similarly no correlation was found between the $s$-process elements measured here and the corresponding $\mathrm{Na}$ abundance measured in $\mathrm{C} 09$. This is seen in Figs. 15a and $\mathrm{c}$ where both $\mathrm{Na}$ and $\mathrm{Ba}$ are highly dispersed for both the $\mathrm{W} 13$ and DO10 $\mathrm{W}_{\mathrm{W} 13}$ samples.

Because the $\mathrm{Na}$ and $\mathrm{Ba}$ do not correlate then these elements were produced by different sources which polluted the medium from which the observed stars formed, as neither Na nor Ba can be produced in these observed stars themselves. Hence this limits the potential AGB polluter to those with sufficient mass to produce $\mathrm{Na}$ but not $\mathrm{Ba}$ (in the thermally pulsing stage) and is evidence for at least two generations of stars in M 15 (Carretta et al. $2009 \mathrm{~b}$ ). This is borne out by comparison to theoretical yields of $s$-process element abundances from AGB models. Lugaro et al. (2012) present $s$-process yields calculated from AGB models at $[\mathrm{Fe} / \mathrm{H}]=-2.3 \mathrm{dex}$, comparable to the metallicity of M 15. The expected $[\mathrm{Ba} / \mathrm{Eu}]$ abundances for a range of stellar masses all exceed $[\mathrm{Ba} / \mathrm{Eu}]=0.0$ dex (see Tables 3 and 4 of that paper). This is therefore in support of the conclusion here that any pollution from AGB in M 15 contributed only to the light element abundances, not to the $s$-process element abundances.

The Na-O anticorrelation is a well-studied characteristic of GCs (Carretta et al. 2010, and references therein). It is proposed that the first generation of stars inherited the signature of the supernova nucleosynthesis whereby the $\mathrm{O}$ abundances are enhanced to above solar values while the $\mathrm{Na}$ is depleted below solar values. During the lifetimes of the massive stars of this population the material is processed in hot H-burning such that the $\mathrm{O}$ is depleted and the $\mathrm{Na}$ is enhanced. This level of depletion and enhancement depends on the characteristics of the individual stars. Hence a continuum is seen for the $\mathrm{Na}-\mathrm{O}$ anticorrelation as different levels of contribution are made to the second generation star-forming material depending on the contributing first generation star. Therefore the second generation of stars bear the signature of the nucleosynthetic processing that occurred within the first generation stars.
Figure $15 \mathrm{~b}$ and e show the Na-O distribution for the $\mathrm{W} 13$ and $\mathrm{DO} 10_{\mathrm{W} 13}$ samples respectively. The $\mathrm{Na}-\mathrm{O}$ anticorrelation is clear in both samples. Hence evidence of at least two generations of stars in M 15. So where does the Ba bimodal distribution fit in? Does one of the modes represent the first generation and the other the second generation? If so then one mode would show the signature of super-solar $\mathrm{O}$ and sub-solar $\mathrm{Na}$ abundances, while the other is depleted in $\mathrm{O}$ and enhanced in $\mathrm{Na}$.

Figure $15 \mathrm{c}$ and decompose the $\mathrm{Na}-\mathrm{O}$ distribution into the two $\mathrm{Ba}$ modes for $\mathrm{W} 13$ and $\mathrm{DO} 10_{\mathrm{W} 13}$ respectively, as defined in Fig. 15a and c. In both instances we restricted the modes to those stars most likely to be in each mode, and so did not include those stars near the mean of each sample as illustrated by the grey dashed lines in the figures. What is most clearly seen in the W13 sample is that both Ba modes show the anti-correlation in Na-O. In the DO10 ${ }_{\mathrm{W} 13}$ sample the anticorrelation is clear for Mode I but more truncated in Mode II. The two modes are less evenly populated for $\mathrm{DO} 10_{\mathrm{W} 13}$, as was seen in Fig. 12 and so the sample size for Mode II may be insufficient.

Based on the W13 sample, the polluters of Mode II appear to have undergone more processing of the material because the correlation extends further into the enhanced $\mathrm{Na}$, depleted $\mathrm{O}$ regime.

Taking the two Ba modes and then the two stellar generations within each mode there is a potential scenario for the formation of M 15 which involves four populations of stars:

- Mode I: primordial showing SN enrichment (relatively poor $\mathrm{Ba}$ and $\mathrm{Eu}$, sub-solar $\mathrm{Na}$, super-solar $\mathrm{O}$ );

- Mode I: second generation showing SN enrichment and $\mathrm{H}$-burning products (relatively poor $\mathrm{Ba}$ and $\mathrm{Eu}$, enhanced $\mathrm{Na}$, depleted O);

- Mode II: primordial showing SN enrichment (relatively rich $\mathrm{Ba}$ and $\mathrm{Eu}$, sub-solar $\mathrm{Na}$, super-solar $\mathrm{O}$ );

- Mode II: second generation showing SN enrichment and $\mathrm{H}$-burning products (relatively rich $\mathrm{Ba}$ and $\mathrm{Eu}$, enhanced $\mathrm{Na}$, depleted O).

In such a scenario each mode was initially polluted by the explosive nucleosynthesis of massive stars with slightly different heavy element yields resulting in the bimodal distribution that has been observed in the W13 sample. This difference in composition may have had the follow on effect of greater processing of the $\mathrm{O}$ to $\mathrm{Na}$ for the higher $\mathrm{Ba}$ mode (II). Integral to formulating such a scenario are the timescales that have been involved. Did these two modes evolve simultaneously, and somehow disconnectedly, either separately or within the same cluster space, or did one population evolve followed by the other?

Alternatively these signatures could reflect localised events. The stars all formed on the same timescale but the initial primordial material was not well-mixed such that stars in one region were more enhanced (depleted) in the heavy elements depending on the level of polllution by the SN. If the bimodal distribution is real then it would appear that the $\mathrm{SN}$ events were restricted to two types. If the bimodal distribution is not real, and there is a continuum of heavy element abundances, as might be argued based on the DO10 ${ }_{\mathrm{W} 13}$ sample, then this restriction is relaxed and the Na-O anticorrelation may be representative of a single mode scenario involving a single primordial population and a single second generation population.

Sk11 investigated the heavy element abundances for 7 RHB and 3 RGB stars in M 15, in combination with a re-analysis of the S97 stars using the improved analysis techniques, and found no evidence of a bimodal distribution in $\mathrm{Ba}$ and $\mathrm{Eu}$ for the sample. Detailed investigation was given into light as well as heavy 
a) W12

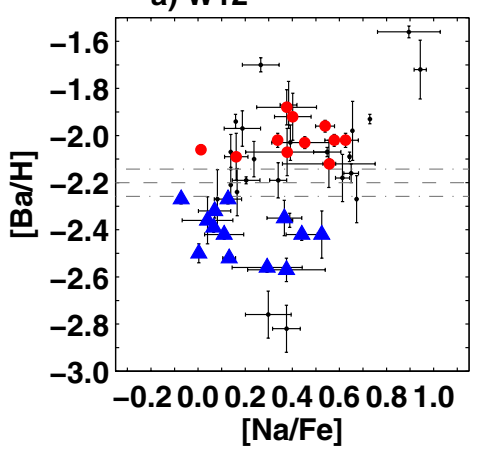

d) $\mathrm{DO10}$

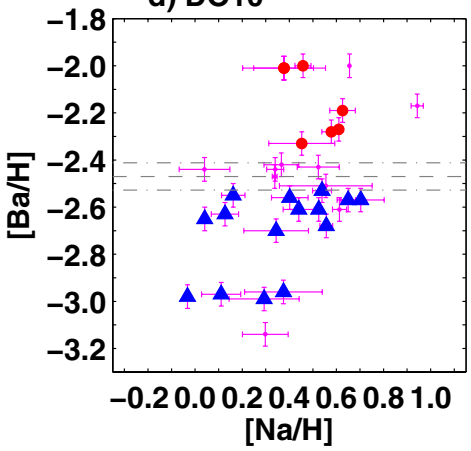

b) W12

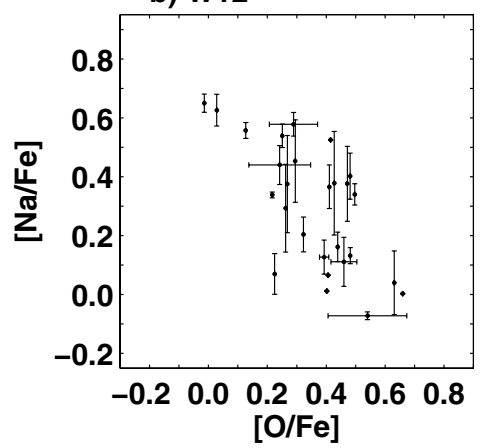

e) D010

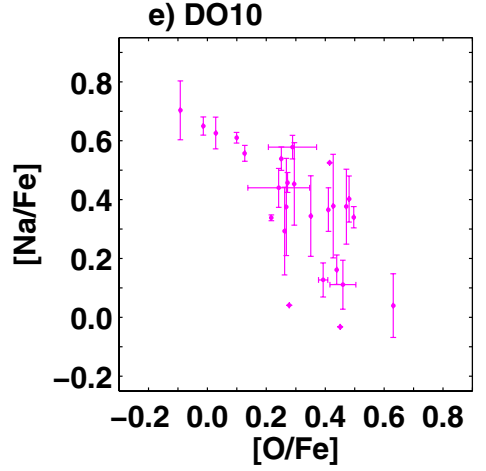

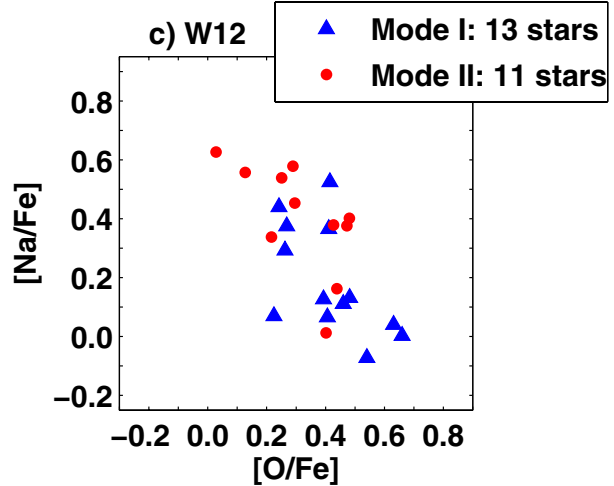

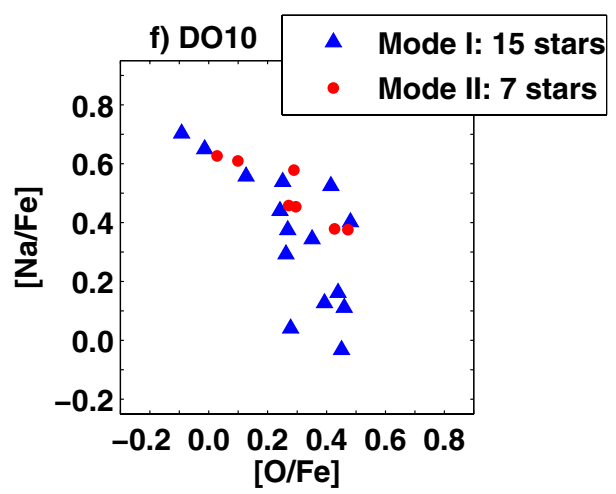

Fig. 15. a) $[\mathrm{Ba} / \mathrm{Eu}]$ against $[\mathrm{Na} / \mathrm{Fe}]$ for 49 stars in the W13 sample (black). Dashed grey line indicates the W13 mode threshold. Dash-dotted grey lines indicates the mean $\sigma[\mathrm{Ba} / \mathrm{H}]$ above and below the threshold. Blue triangles identify those clearly in Mode I, red dots are those clearly in Mode II. b) $[\mathrm{Na} / \mathrm{Fe}]$ against $[\mathrm{O} / \mathrm{Fe}]$ for the $\mathrm{W} 13$ stars with both measurements. c) As for b) but the red and blue points from a). d) to f) are as for a) to c) but for $\mathrm{DO} 10_{\mathrm{W} 13}$ (magenta).

$s$-process elements whereby the signatures of the 10 key stars showed evidence of enrichment in the heavy elements that was not attributable to either the $r$-nor $s$-process but potentially due to a LEPP. Due to the wavelength range of the observations analysed in the current paper there were no spectral features of any light $s$-process elements $(\mathrm{Sr}, \mathrm{Y}, \mathrm{Zr}$ ) present, hence this line of research could not be pursued.

The above investigation of the $s$-process residual with $[\mathrm{Ba} / \mathrm{H}],[\mathrm{La} / \mathrm{H}]$ and $[\mathrm{Eu} / \mathrm{H}]$ confirmed that both modes are dominated by contributions from the $r$-process, hence pollution by explosive nucleosynthesis in massive stars. Some small contribution to the heavy element abundances by another process cannot be discounted as the residuals for both modes lie above the $r$-process only yield limit, although predictions of heavy element yields from explosive nucleosynthesis have their own set of uncertainties. As such the distribution of heavy elements in this sample of M 15 giant stars implies one mode that is metal poor with low $\mathrm{Ba}, \mathrm{La}$ and $\mathrm{Eu}$ abundances, and a second mode which is slightly less metal poor $(\Delta[\mathrm{Fe} / \mathrm{H}]=+0.03 \mathrm{dex})$ with relatively higher $\mathrm{Ba}, \mathrm{La}$ and $\mathrm{Eu}$ abundances that all have similar dispersions on the order of the dispersion for Fe.

In effect the analysis carried out here has drawn the same conclusions as S97, S00a and S00b that there is a bimodal distribution in the $\mathrm{Ba}, \mathrm{Eu}$, and now potentially $\mathrm{La}$, abundances in M 15 giant stars and that both modes therefore have a heavy element enrichment history that is dominated by the $r$-process. The addition of the $\mathrm{Na}-\mathrm{O}$ abundances from $\mathrm{C} 09$ have added another layer of information which potentially describes a very complex formation scenario for this very interesting GC.

Throughout Figs. 11 and 13 there is an outlier (C09 ID 42262) in the high Ba Mode that is enhanced in Ba and La to a level which is significantly greater than the rest of the W13 stars. However, the Eu abundance is fairly typical of that mode. The $s$-process residuals for this star place it close to the solar value. Given the conclusions above and that because this is an RGB star it cannot be producing the heavy elements internally, the most likely scenario is that this star has been the recipient of mass transfer from a companion star that has resulted in the observed enhancments. The fact that the star is not further enhanced in Eu (the $r$-process element) and both $\mathrm{Ba}$ and La are heavy $s$-process elements implies that the polluting star had evolved through the thermally pulsing AGB stage.

One other star (C09 ID 37931) in the W13 sample is similarly enhanced in Ba but no La abundance could be measured. This star is also enhanced in Eu, albeit with large uncertainties. There are potentially two such stars within the S97 sample as well.

\section{Conclusions}

This is the largest study of barium in giant stars in M 15 todate. The sample, based on the original programme in $\mathrm{C} 09$, is well-sampled along the RGB. La and Eu abundances were determined for a subset of this sample furthering this investigation into heavy element abundances in M 15. In parallel the equivalent width measurements from DO10 and S97 were re-analysed on the C09 scale which provided an interesting comparison for key element distributions.

The stellar parameters for these objects were take from C09, except for $\xi$ which was re-determined based on a $\xi$-log $g$ relation derived from a subsample of stars for which a reasonable sample of Fe I lines could be measured. The key Ba line under analysis was shown to be highly sensitive to $\xi$ and so a great effort was made to reduce the associated errors.

A bimodal distribution of $\mathrm{Ba}, \mathrm{Eu}$, and potentially $\mathrm{La}$, was found in the W13 sample in agreement with previous studies. 
Based on the W13 results, an examination of the $r$ - and $s$-process contributions to these heavy element abundances leads to the two modes having similar pollution scenarios of a single burst of explosive nucleosynthesis by massive stars (for example, Type II supernova) that enriched the interstellar medium from which the stars in each mode formed. The enrichment was to different degrees between the modes.

Further enrichment by another process that produces heavy elements can not be discounted but the wavelength region observed here did not allow for analysis of other heavy element features.

For both modes a Na-O anticorrelation was observed which is indicative of two stellar generations in each mode: a primodial and second generation in Mode I, and a primordial and second generation in Mode II. A scenario that can account for such a set of stellar populations is necessarily complex. If the Ba distribution is more indicative of a continuum of $\mathrm{Ba}$ and $\mathrm{Eu}$ abundances then a single mode of stellar evolution could explain the observed $\mathrm{Na}-\mathrm{O}$ anticorrelation.

While this study strongly supports the argument for a bimodal distribution in $\mathrm{Ba}, \mathrm{La}$ and $\mathrm{Eu}$ for $\mathrm{M} 15$, there are inherent uncertainties in the measurement of the key $\mathrm{Ba}$ line due to its sensitivity to $\xi$. La would be a better candidate as it has no such sensitivites. Hence re-observing the entire sample considered here at higher resolution and $\mathrm{S} / \mathrm{N}$ in order to complete the $\mathrm{La}$ and $\mathrm{Eu}$ abundances for the sample would prove definitive. Extending the observed wavelength regions to include features of the light $s$-process peak (Y, Sr, $\mathrm{Zr}$ ) would enrich the analysis as to the potential contribution from other heavy element processes.

Acknowledgements. This research has made use of the SIMBAD database, operated at CDS, Strasbourg, France.

\section{References}

Alonso, A., Arribas, S., \& Martínez-Roger, C. 1999, A\&AS, 140, 261 Andrievsky, S. M., Spite, M., Korotin, S. A., et al. 2009, A\&A, 494, 1083 Busso, M., Gallino, R., Lambert, D. L., Travaglio, C., \& Smith, V. V. 2001, ApJ, 557,802

Carretta, E., Bragaglia, A., Gratton, R., \& Lucatello, S. 2009a, A\&A, 505, 139

Carretta, E., Bragaglia, A., Gratton, R. G., et al. 2009b, A\&A, 505, 117

Carretta, E., Bragaglia, A., Gratton, R. G., et al. 2010, A\&A, 516, A55

Chen, Z., Zhang, J., Chen, Y., Cui, W., \& Zhang, B. 2006, Ap\&SS, 306, 33

Davidson, M. D., Snoek, L. C., Volten, H., \& Doenszelmann, A. 1992, A\&A, 255,457

D’Orazi, V., Gratton, R., Lucatello, S., et al. 2010, ApJ, 719, L213

Edvardsson, B., Andersen, J., Gustafsson, B., et al. 1993, A\&A, 275, 101

Gratton, R. G., Carretta, E., \& Bragaglia, A. 2012, A\&ARv, 20, 50

Iliadis, C. 2007, Nuclear Physics of Stars (Wiley-VCH)

Kirby, E. N., Guhathakurta, P., Bolte, M., Sneden, C., \& Geha, M. C. 2009, ApJ, 705,328

Lawler, J. E., Bonvallet, G., \& Sneden, C. 2001a, ApJ, 556, 452

Lawler, J. E., Wickliffe, M. E., den Hartog, E. A., \& Sneden, C. 2001b, ApJ, 563,1075

Lodders, K. 2003, ApJ, 591, 1220

Lugaro, M., Karakas, A. I., Stancliffe, R. J., \& Rijs, C. 2012, ApJ, 747, 2

Otsuki, K., Honda, S., Aoki, W., Kajino, T., \& Mathews, G. J. 2006, ApJ, 641, L117

Preston, G. W., Sneden, C., Thompson, I. B., Shectman, S. A., \& Burley, G. S. 2006, AJ, 132, 85

Roederer, I. U. 2011, ApJ, 732, L17

Rutten, R. J. 1978, Sol. Phys., 56, 237

Short, C. I., \& Hauschildt, P. H. 2006, ApJ, 641, 494

Simmerer, J., Sneden, C., Cowan, J. J., et al. 2004, ApJ, 617, 1091

Sneden, C. 1973, Ph.D. Thesis, University of Texas at Austin

Sneden, C., Kraft, R. P., Shetrone, M. D., et al. 1997, AJ, 114, 1964

Sneden, C., Johnson, J., Kraft, R. P., et al. 2000a, ApJ, 536, L85

Sneden, C., Pilachowski, C. A., \& Kraft, R. P. 2000b, AJ, 120, 1351

Sneden, C., Cowan, J. J., \& Gallino, R. 2008, ARA\&A, 46, 241

Sobeck, J. S., Kraft, R. P., Sneden, C., et al. 2011, AJ, 141, 175

Worley, C. C., de Laverny, P., Recio-Blanco, A., et al. 2012, A\&A, 542, A48

Pages 17 to 20 are available in the electronic edition of the journal at http://www . aanda. org 
C. C. Worley et al.: Ba and Eu abundances in M 15 giant stars

Table 1. Star ID, effective temperature, surface gravity, model metallicity, microturbulence, radial velocity, abundances derived from Fe I and $\mathrm{Fe}$ II lines, $\mathrm{Na}$, and $\mathrm{O}$ abundances and their associated root mean square error (rms) for each of the M 15 stars analysed here as determined in C09.

\begin{tabular}{|c|c|c|c|c|c|c|c|c|c|c|c|c|c|}
\hline ID & $\begin{array}{l}T_{\text {eff }} \\
(\mathrm{K})\end{array}$ & $\begin{array}{l}\log g \\
(\operatorname{dex})\end{array}$ & $\begin{array}{c}{[\mathrm{A} / \mathrm{H}]} \\
(\mathrm{dex})\end{array}$ & $\begin{array}{c}\xi \\
\left(\mathrm{km} \mathrm{s}^{-1}\right)\end{array}$ & $\begin{array}{c}V_{\mathrm{rad}} \\
\left(\mathrm{km} \mathrm{s}^{-1}\right)\end{array}$ & $\begin{array}{c}{[\mathrm{Fe} \mathrm{I} / \mathrm{H}]} \\
(\operatorname{dex})\end{array}$ & rms & $\begin{array}{c}{[\mathrm{Fe} \text { II } / \mathrm{H}]} \\
(\mathrm{dex})\end{array}$ & $\mathrm{rms}$ & $\begin{array}{c}{[\mathrm{O} / \mathrm{Fe}]} \\
(\mathrm{dex})\end{array}$ & rms & $\begin{array}{c}{[\mathrm{Na} / \mathrm{Fe}]} \\
(\mathrm{dex})\end{array}$ & rms \\
\hline 40825 & 4313 & 0.65 & -2.33 & 2.25 & -113.91 & -2.33 & 0.14 & -2.34 & - & 0.24 & 0.11 & 0.44 & 0.07 \\
\hline 4099 & 4324 & 0.69 & -2.32 & 2.03 & -109.43 & -2.32 & 0.11 & & & $0.54^{*}$ & 0.13 & $-0.07^{*}$ & 0.01 \\
\hline 43788 & 4325 & 0.52 & -2.36 & 2.40 & -96.93 & -2.36 & 0.10 & -2.46 & 0.08 & 0.39 & 0.02 & 0.13 & 0.06 \\
\hline 31914 & 4338 & 0.58 & -2.20 & 2.09 & -112.77 & -2.20 & 0.10 & -2.21 & - & -0.32 & - & & \\
\hline 41287 & 4373 & 0.78 & -2.36 & 1.10 & -116.25 & -2.36 & 0.07 & & & & & 0.39 & 0.01 \\
\hline 34519 & 4416 & 0.87 & -2.38 & 1.62 & -98.04 & -2.38 & 0.11 & -2.46 & - & 0.46 & 0.04 & 0.11 & 0.08 \\
\hline 37215 & 4445 & 0.98 & -2.31 & 1.28 & -111.36 & -2.31 & 0.01 & & & & & 0.73 & - \\
\hline 34995 & 4468 & 1.00 & -2.34 & 0.79 & -115.14 & -2.34 & 0.08 & & & & & 0.64 & - \\
\hline 3137 & 4486 & 1.08 & -2.35 & 2.27 & -109.50 & -2.35 & 0.13 & -2.29 & - & 0.29 & 0.08 & 0.58 & 0.04 \\
\hline 42262 & 4528 & 1.13 & -2.33 & 2.50 & -104.50 & -2.33 & 0.08 & & & & & 0.90 & 0.13 \\
\hline 26751 & 4533 & 1.18 & -2.44 & 1.04 & -98.72 & -2.44 & 0.08 & & & & & 0.55 & 0.06 \\
\hline 38678 & 4554 & 1.23 & -2.35 & 1.28 & -109.01 & -2.35 & 0.08 & -2.46 & 0.01 & 0.13 & - & 0.56 & 0.03 \\
\hline 2792 & 4567 & 1.26 & -2.32 & 1.50 & -100.93 & -2.32 & 0.13 & & & $0.32^{*}$ & 0.00 & $0.20^{*}$ & 0.06 \\
\hline 39752 & 4598 & 1.31 & -2.36 & 1.30 & -108.38 & -2.36 & 0.13 & -2.39 & 0.03 & 0.26 & - & 0.29 & 0.15 \\
\hline 34456 & 4621 & 1.37 & -2.34 & 1.40 & -97.02 & -2.34 & 0.20 & & & 0.22 & - & 0.34 & 0.01 \\
\hline 34961 & 4627 & 1.41 & -2.32 & 1.85 & -113.28 & -2.32 & 0.15 & -2.36 & 0.02 & -0.01 & - & 0.65 & 0.03 \\
\hline 39787 & 4630 & 1.35 & -2.43 & 2.10 & -111.94 & -2.43 & 0.13 & & & $0.48^{*}$ & 0.00 & $0.13^{*}$ & 0.03 \\
\hline 38329 & 4664 & 1.44 & -2.37 & 0.86 & -109.04 & -2.37 & 0.02 & & & & & 0.16 & - \\
\hline 45062 & 4700 & 1.54 & -2.31 & 1.66 & -110.27 & -2.31 & 0.11 & -2.23 & - & 0.03 & - & 0.63 & 0.05 \\
\hline 20498 & 4717 & 1.61 & -2.49 & 1.12 & -106.58 & -2.49 & 0.08 & -2.44 & - & 0.25 & - & 0.54 & 0.04 \\
\hline 21948 & 4746 & 1.66 & -2.49 & 0.20 & -108.68 & -2.49 & 0.05 & & & & & 0.46 & - \\
\hline 28510 & 4754 & 1.64 & -2.42 & 0.14 & -100.52 & -2.42 & 0.02 & & & $0.23^{*}$ & 0.00 & $0.07^{*}$ & 0.07 \\
\hline 29264 & 4810 & 1.77 & -2.35 & 0.84 & -101.86 & -2.35 & 0.14 & & & $0.40^{*}$ & 0.00 & $0.01^{*}$ & 0.00 \\
\hline 18815 & 4832 & 1.83 & -2.29 & 0.80 & -108.68 & -2.29 & 0.10 & & & $0.66^{*}$ & 0.00 & $0.00^{*}$ & 0.00 \\
\hline 42362 & 4952 & 2.03 & -2.41 & 1.00 & -110.67 & -2.41 & 0.09 & & & 0.27 & - & 0.38 & 0.17 \\
\hline 29480 & 4968 & 2.10 & -2.33 & 1.16 & -104.60 & -2.33 & 0.16 & & & 0.30 & - & 0.45 & 0.14 \\
\hline 37931 & 4976 & 2.10 & -2.37 & 1.21 & -111.25 & -2.37 & 0.05 & & & & & 0.27 & 0.08 \\
\hline 31791 & 4978 & 2.13 & -2.28 & 0.10 & -104.92 & -2.28 & 0.03 & & & & & 0.14 & - \\
\hline 33484 & 5036 & 2.22 & -2.44 & 1.20 & -109.75 & -2.44 & 0.17 & & & & & 0.14 & - \\
\hline 10329 & 5060 & 2.25 & -2.38 & 1.25 & -109.91 & -2.38 & 0.11 & & & 0.42 & - & 0.53 & - \\
\hline 27889 & 5063 & 2.28 & -2.32 & 1.15 & -101.14 & -2.32 & 0.13 & & & 0.63 & - & 0.04 & 0.11 \\
\hline 39741 & 5076 & 2.31 & -2.25 & 1.42 & -112.10 & -2.25 & 0.20 & & & 0.43 & - & 0.38 & 0.18 \\
\hline 42674 & 5076 & 2.29 & -2.43 & 1.35 & -96.28 & -2.43 & 0.06 & & & & & 0.67 & - \\
\hline 17458 & 5081 & 2.33 & -2.31 & 0.92 & -110.56 & -2.31 & 0.19 & & & 0.44 & - & 0.16 & 0.05 \\
\hline 40086 & 5087 & 2.36 & -2.32 & 1.48 & -122.86 & -2.32 & 0.10 & & & 0.41 & - & 0.37 & 0.07 \\
\hline 36274 & 5105 & 2.35 & -2.35 & 1.16 & -100.30 & -2.35 & 0.13 & & & 0.50 & - & 0.34 & 0.04 \\
\hline 26759 & 5106 & 2.34 & -2.35 & 1.16 & -101.07 & -2.35 & 0.20 & & & & & 0.24 & - \\
\hline 29521 & 5129 & 2.46 & -2.30 & 0.03 & -101.45 & -2.30 & 0.02 & & & & & 0.19 & 0.08 \\
\hline 41987 & 5130 & 2.41 & -2.33 & 2.20 & -108.07 & -2.33 & 0.09 & & & & & 0.39 & - \\
\hline 1939 & 5149 & 2.48 & -2.41 & 0.88 & -101.98 & -2.41 & 0.03 & & & 0.32 & - & & \\
\hline 34272 & 5151 & 2.47 & -2.25 & 1.24 & -108.35 & -2.25 & 0.10 & & & 0.47 & - & 0.38 & 0.13 \\
\hline 35961 & 5156 & 2.44 & -2.41 & 0.40 & -107.90 & -2.41 & 0.10 & & & & & 0.38 & 0.06 \\
\hline 31010 & 5164 & 2.46 & -2.32 & 0.66 & -115.45 & -2.32 & 0.08 & & & & & 0.61 & 0.03 \\
\hline 23216 & 5216 & 2.57 & -2.32 & 0.14 & -104.01 & -2.32 & 0.02 & & & & & & \\
\hline 18240 & 5229 & 2.59 & -2.30 & 0.94 & -103.56 & -2.30 & 0.03 & & & & & 0.56 & 0.20 \\
\hline 32942 & 5236 & 2.61 & -2.25 & 1.82 & -108.90 & -2.25 & 0.10 & & & & & 0.38 & 0.04 \\
\hline 32979 & 5236 & 2.57 & -2.35 & 1.97 & -106.56 & -2.35 & 0.04 & & & & & 0.17 & - \\
\hline 2411 & 5239 & 2.65 & -2.29 & 1.07 & -110.01 & -2.29 & 0.22 & & & & & & \\
\hline 23153 & 5242 & 2.59 & -2.26 & 1.61 & -108.68 & -2.26 & 0.10 & & & & & & \\
\hline 8927 & 5250 & 2.64 & -2.42 & 0.16 & -104.84 & -2.42 & 0.08 & & & & & 0.30 & 0.10 \\
\hline 18770 & 5260 & 2.63 & -2.38 & 1.95 & -103.56 & -2.38 & 0.10 & & & & & & \\
\hline 22441 & 5270 & 2.70 & -2.35 & 0.35 & -108.68 & -2.35 & 0.09 & & & & & & \\
\hline 18508 & 5276 & 2.68 & -2.26 & 2.61 & -103.56 & -2.26 & 0.09 & & & & & & \\
\hline 9608 & 5291 & 2.68 & -2.33 & 0.31 & -104.47 & -2.33 & 0.12 & & & 0.41 & - & & \\
\hline 29436 & 5312 & 2.81 & -2.41 & 1.39 & -98.38 & -2.41 & 0.11 & & & & & & \\
\hline 4989 & 5319 & 2.72 & -2.38 & 0.10 & -104.70 & -2.38 & 0.06 & & & & & & \\
\hline 3825 & 5323 & 2.76 & -2.26 & 0.72 & -109.12 & -2.26 & 0.12 & -2.24 & 0.34 & & & & \\
\hline 40762 & 5325 & 2.78 & -2.34 & 1.17 & -115.00 & -2.34 & 0.07 & -2.21 & - & & & 0.66 & - \\
\hline 8329 & 5344 & 2.86 & -2.41 & 1.77 & -106.98 & -2.41 & 0.02 & & & & & & \\
\hline 2463 & 5349 & 2.84 & -2.31 & 1.01 & -105.89 & -2.31 & 0.12 & -2.27 & - & 0.48 & - & 0.40 & 0.08 \\
\hline 19346 & 5349 & 2.80 & -2.34 & 2.70 & -105.99 & -2.34 & 0.04 & & & & & 0.94 & 0.03 \\
\hline 28026 & 5362 & 2.81 & -2.38 & 1.80 & -111.48 & -2.38 & 0.15 & & & & & & \\
\hline 31796 & 5374 & 2.87 & -2.25 & 1.80 & -110.52 & -2.25 & 0.08 & & & & & 0.08 & - \\
\hline
\end{tabular}

Notes. ${ }^{(*)}[\mathrm{O} / \mathrm{Fe}] \&[\mathrm{Na} / \mathrm{Fe}]$ taken from Carretta et al. (2009a). 
Table 3. Hyperfine structure decomposition based on atomic information obtained from the associated references for the Ba II (Rutten 1978; Davidson et al. 1992), La II (Lawler et al. 2001a) and Eu II (Lawler et al. 2001b) spectral lines used in the spectrum synthesis of the M 15 giant stars.

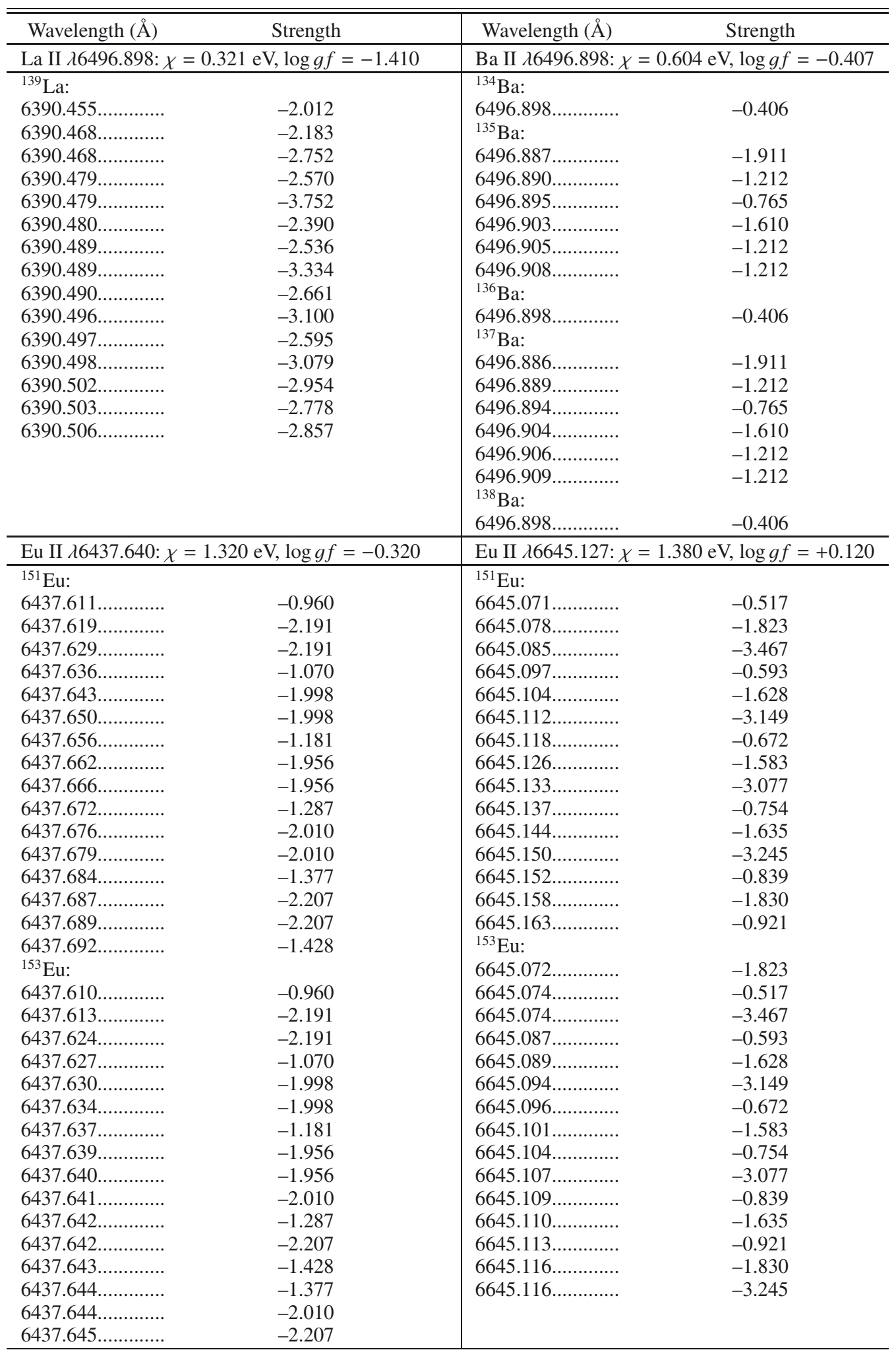




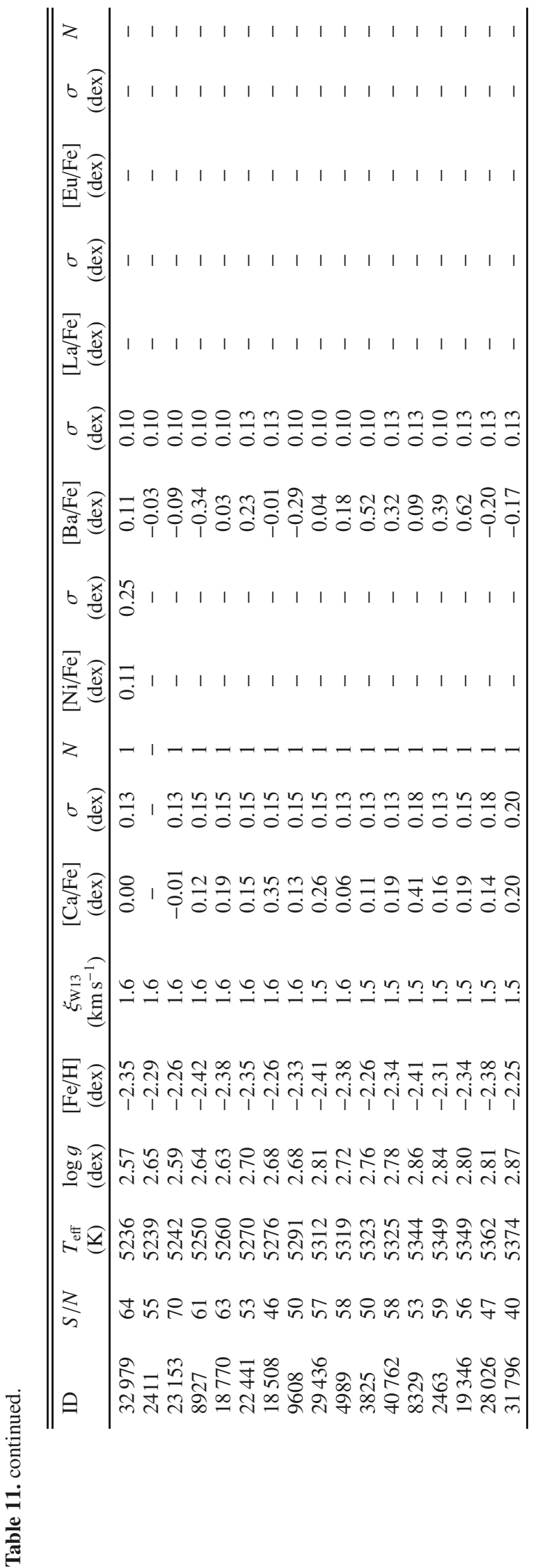

